\title{
Electrophysiological evidence that psychopathic personality traits are associated with atypical response to salient distractors
}

\author{
Patrick L. Carolan ${ }^{1,2} \cdot$ John M. Gaspar ${ }^{1} \cdot$ Killian Kleffner $^{1} \cdot$ Mario Liotti $^{1}$
}

Published online: 2 January 2020

(C) The Psychonomic Society, Inc. 2020

\begin{abstract}
Event-related potentials (ERPs) were used to assess the neural mechanisms underlying visual-spatial attention abnormalities associated with psychopathic personality traits. Sixty-nine undergraduates (56 women, 13 men) completed the Psychopathic Personality Inventory-Revised (PPI-R; Lilienfeld \& Widows, 2005) and performed two cognitive tasks in which search displays containing a lateralized singleton encircled a fixation point that changed luminance from trial-to-trial. When searching for the singleton as a target, PPI-R scores were uncorrelated with ERP measures of its salience (Ppc), goal-directed selection (N2pc), and working memory evaluation (negative amplitude CDA). In contrast, when responding to the changes in luminance at fixation and ignoring the lateral singleton as a salient distractor, PPI-R Self-Centered Impulsivity factor scores were positively correlated with a potential indicator of distractor suppression (a sustained positive amplitude CDA). These findings provide support for a neurophysiological interpretation of the changes in visual-spatial attention associated with psychopathic personality traits: normal selection of target information accompanied by greater elimination of distractor information at a later visual working memory stage.
\end{abstract}

Keywords Psychopathic personality traits $\cdot$ Event-related potentials $\cdot \mathrm{N} 2 \mathrm{pc} \cdot$ Contralateral delay activity $\cdot$ Posterior contralateral positivity $\cdot$ Distractor positivity

Psychopathic personality is characterized by deficits in emotional capacity and disinhibited socially-deviant behavior (Lilienfeld \& Widows, 2005). In extreme presentations, it is a notable predictor of instrumental criminal violence. Such presentations are rare, however, and psychopathy often is conceptualized as a conflux of traits that are otherwise normally distributed throughout both community and correctional populations (Benning, Patrick, Hicks, Blonigen, \& Krueger, 2003). Clinical assessments, such as the Psychopathic Personality Checklist-Revised (PCL-R; Hare, 2003), and

General Scientific Summary This research indicates that when trying to focus attention, psychopathic personality traits are associated with heightened suppression of distractors, rather than improved selection of targets.

Patrick L. Carolan

patrick.carolan@smu.ca

1 Department of Psychology, Simon Fraser University, Burnaby, Canada

2 Department of Psychology, Saint Mary's University, 923 Robie Street, Halifax, NS B3H 3C3, Canada self-report assessments, such as the Psychopathic Personality Inventory-revised (PPI-R; Lilienfeld \& Widows, 2005), cluster these traits into factors that broadly capture blunted emotionality, callousness, egocentricity, and impulsivity. As these factors independently predict scores on measures of affect, impulsivity, drive, and inhibition, psychopathic personality has an undeniably complex psychometric profile, or nomological web (Kastner, Sellbom, \& Lilienfeld, 2012; Ray, Weir, Poythress, \& Rickelm, 2011; Uzieblo, Verschuere, Van den Bussche, \& Crombez, 2010). However, the actual neural mechanisms that underlie these executive and emotional characteristics remain unclear.

The response modulation theory posits that an early attention bottleneck limits the propensity of individuals with psychopathy to engage in inhibitory control and affective response (Baskin-Sommers, Curtin, Li, \& Newman, 2012; Glass \& Newman, 2006; Newman \& Baskin-Sommers, 2012; Patterson \& Newman, 1993; Wolf et al., 2012). When such individuals establish an attention set directed towards accomplishing a particular goal, they fail to shift focus to external information that might otherwise be important. This rigidity can result in response perseveration even in the face of important inhibitory cues, such as punishment contingencies 
or emotional expressions (Newman \& Kosson, 1986). Behavioral evidence of such a bottleneck in psychopathic personality has been demonstrated with the attentional blink task, a canonical measure of selective attention for temporally separated but spatial invariant information. Specifically, compared with nonpsychopathic inmates, attentional blink interference was found to be attenuated in inmates classified as psychopathic using the PCL-R (Wolf et al., 2012). Behavioral data also has suggested that visual-spatial selective attention is impacted by this bottleneck in psychopathic personality. For example, in a color-word Stroop study conducted with an offender sample, Hiatt, Schmitt, and Newman (2004) found that inmates classified as psychopathic with the PCL-R showed less Stroop interference than those rated as nonpsychopathic but only if color and semantic information were spatially separated. In a follow-up study, inmates classified as psychopathic showed less distractor interference than nonpsychopathic inmates during a modified Erikson Flanker task if a cue directed attention to the target's location (Zeier, Maxwell, \& Newman, 2009). Thus, the response modulation theory holds that although inhibitory capacity per se is not impaired in psychopathy, the stimuli that should otherwise inhibit responses simply go unattended when they fall outside immediate spatial attention.

An emerging body of research provides neurological evidence of the attention-mediated reduction in emotional responding proposed in the response modulation theory. For example, several electroencephalographic (EEG) studies have reported that event-related potential (ERP) indices of threat response and emotional interference are blunted in criminal and noncriminal individuals high in psychopathic traits when they view stimuli without directly attending to threat-relevant or affective dimensions (Anderson \& Stanford, 2012; BaskinSommers et al., 2012; Carolan, Jaspers-Fayer, Asmaro, Douglas, \& Liotti, 2014; Medina, Kirilko, \& Grose-Fifer, 2016). However Wolf et al. (2012) have identified the need to situate the response modulation theory's attention bottleneck proposal within the broader context of established and canonical attention measures. Furthermore, despite the compelling behavioral evidence of a visual-spatial bottleneck in psychopathic personality, the neural mechanisms underlying this effect have received considerably less research focus. One ERP replication of Hiatt et al.'s (2004) spatially separated colorword Stroop task with an offender sample did find that psychopathic personality moderated the association between interference and an early fronto-central negativity (N100; Hamilton, Baskin-Sommers, \& Newman, 2014). The authors of this study noted the smaller N100 of high PCL-R inmates on emotion incongruent trials might reflect their reduced tendency to allocate attention to salient but task irrelevant information. However this study is only a first step toward situating the attention bottleneck proposal within a much larger visualspatial attention research field: visual search. In the current study, we further link these two fields by performing an EEG investigation of psychopathic personality traits conducted within a visual search testing framework, using an undergraduate sample assessed with the PPI-R (Lilienfeld \& Widows, 2005; see Methods for a detailed description of this scale).

Visual search — the act of locating a target within a group of distractors - is an everyday task that highlights several fundamental visual-spatial mechanisms and demonstrates that attention is not a unitary phenomenon (Di Lollo, Enns, \& Rensink, 2000; Luck \& Kappenman, 2013). Instead, the term attention broadly encompasses a suite of nervous system processes that collectively focus cognition, perception, and multiple sensory modalities into high-resolution representations of select mental or environmental phenomena, while simultaneously encoding low-resolution representations of ambient stimuli (Wolfe \& Horowitz, 2004). This coupling maximizes signal processing efficacy for central-events, while still allowing peripheral-events some limited access to the "mind's eye" lest they become relevant. Within the context of visual search, the critical mechanisms under consideration include enhancing representations of visual-spatial locations occupied by targets to facilitate their subsequent identification (i.e., target selection and target evaluation respectively), suppressing visual-spatial locations occupied by competing items to limit their access to higher-order cognition (i.e., distractor filtering) and biasing visual cortex sensitivity toward task-relevant stimulus characteristics, such as shape and color to prime the ongoing sensory sweeps that guide these visual-spatial actions (i.e., salience computation).

Visual-spatial attention shifts are either overt, when accompanied by a saccade, or covert, when an observer mentally focuses on or suppresses an object's location without performing an actual eye movement (Luck \& Kappenman, 2013). In both cases, visual-spatial shifts are guided by the relative salience of available items (Wolfe \& Horowitz, 2004), which can be quantified as their degree of difference in some dimensional space, for example, chromaticity in the case of color salience (Gaspar \& McDonald, 2014). Highly salient stimuli are those whose degree of difference from others on a particular feature dimension substantially exceeds the threshold required to simply discriminate them. Salient items are singletons when presented within sets of largely identical items, as is typical in highly controlled laboratory investigations of visual search. In many situations, visualspatial attention deployment is a reflexive process (exogenous or "bottom-up") driven toward the most salient item in the environment (Eimer \& Kiss, 2008). The "inherent" salience of an object will vary based on observer characteristics, history, genetic inheritance, and current context. But all things being equal, an item that is distinctly different on one of a limited number of critical "guiding feature dimensions," including onset, motion, orientation, size, color, luminance, and shape, can effectively capture visual-spatial attention, triggering 
shifts of focus to its location to enable its evaluation (Wolfe \& Horowitz, 2004). Alternatively, visual-spatial attention can be directed intentionally (endogenous or "top-down") to achieve internally generated goals. In such instances, the observer focuses on the location of a task-relevant object to evaluate or identify it. This endogenous search is most efficient when the target is highly salient on a guiding feature (its "signal" is strong), and there is little to no variation amongst the accompanying nontarget items, hereafter referred to as fillers (their "noise" is low). Search may be impeded by the presentence of additional salient nontarget singletons, hereafter referred to as distractors (Gaspar \& McDonald, 2014; Jannati, Gaspar, \& McDonald, 2013).

Despite this distinction between endogenous and exogenous visual-spatial shifts, these processes are interactive. For example, when targets are defined by a particular dimension, such as color, exogenous shifts to any singletons salient on that dimension become more likely due to its task-contingent enhancement. This can result in contingent capture of attention by irrelevant items beyond the capacity predicted by their inherent salience (Eimer \& Kiss, 2008; Hopfinger \& Ries, 2005). As an explanation of these interactive mechanisms, Jannati et al. (2013) have proposed a model in which ongoing visual scans of the environment continually update contextualized salience maps. In turn, these salience maps guide reflexive (exogenous) orienting to stimuli. Executive processes can also direct visual-spatial focus to or from object-locations coded within that salience map in order to perform goal related identification (endogenous attention in the most classic sense). Additionally, endogenous realignment of feature dimension weights used for salience map calculations can increase or decrease the relative salience of objects, and by extension their capacity to guide reflexive and intentional attention shifts in real-time. Once oriented to, an object can then be subject to higher-order identification or discrimination.

By using EEG in laboratory tasks, the processes of target selection, distractor suppression, and item salience computation can each be measured separately with specific ERP components. This enables researchers to determine the cortical mechanisms that mediate differences in observed behaviors. We examined the covariance of PPI-R scores with four of these ERP components. The goal of this investigation was to provide a comprehensive neurobiological account of the visual-spatial abnormalities associated with the traits captured by this measure, as they pertain to the response modulation theory of psychopathic personality.

\section{Lateralized ERP indices of visual-spatial attention mechanisms}

The cross-lateralized structure of the human visual system has facilitated the discovery of several lateralized ERP components indexing specific visual-spatial operations. As noted above, typical ERP visual search investigations employ stimulus arrays containing many identical or largely identical nontarget fillers, as well as salient lateralized singletons that may be distractors or targets depending on task demands. This approach is particularly powerful and dramatically reduces measurement error, because singleton specific activity can be isolated both within-subject and within-trial (Luck, 2014). This is achieved by subtracting stimulus-locked ERP activity of electrodes at scalp locations ipsilateral to (on the same side as) the visual hemifield containing the singleton from ERP activity of electrodes contralateral to (on the opposite side as) the singleton. ${ }^{1}$ Quantitatively, this difference in electrical activity can be tested in an analysis of variance (ANOVA) framework by treating contralateral and ipsilateral sites as two-levels of a within-subjects factor. Alternatively, component amplitudes and latencies can be extracted from contralateral-minusipsilateral difference waveforms and used as outcome variables in correlation and linear regression analyses.

The most widely studied lateralized ERP component is the posterior contralateral N2 (N2pc). The N2pc indexes the strength, timing, and reliability of the visual-spatial selection of a particular item or singleton from a group of stimuli (Eimer \& Kiss, 2010; Luck \& Hillyard, 1994a; Mazza, Turatto, Umiltà, \& Eimer, 2007; McDonald, Green, Jannati, \& Di Lollo, 2013). It is observed from 175-300 ms after stimulus presentation as a greater negativity at singleton contralateral scalp than ipsilateral scalp (Luck \& Hillyard, 1994b). One possible neurobiological explanation of the visual-spatial bottleneck associated with the response modulation theory is that it results from target hyper-selection, which could be indexed by this ERP component. If so, PPI-R scores would be expected to correlate positively with latency or absolute amplitude of the N2pc to a target singleton.

A recent visual search study examined N2pc differences between incarcerated offenders clinically assessed as low, moderate, or high psychopathy using the PCL-R (Krusemark, Kiehl, \& Newman, 2016). For half of the task blocks, the target was distinguished by its size, and in the other half by its color. Critically, all trials were preceded by an array containing an uninformative but salient color probe. Results showed that this probe elicited an N2pc component during color target blocks but not shape target blocks. The phenomenon of an N2pc response to uninformative probes/cues that share features with targets is known as contingent capture and is thought to reflect the impact of an endogenously held attention set (i.e., the goal-directed priming of target features; Eimer \& Kiss, 2008). This effect was strongest amongst the

\footnotetext{
${ }^{1}$ Note: For the remainder of this manuscript "contralateral" refers to electrodes/scalp sites contralateral to the visual hemifield containing the singleton, whereas "ipsilateral" refers to electrodes/scalp sites ipsilateral to the visual hemifield containing the singleton (rather than being contralateral or ipsilateral to some other reference point, such as another electrode).
} 
inmates highest in psychopathic personality, suggesting that psychopathy was associated with exaggerated endogenous attention for the target feature in those blocks, supporting the attention bottleneck proposal. Yet several questions regarding the nature of visual search ERPs in psychopathic personality have yet to be explored. In particular, because only data for probe stimuli were reported in this previous study, it remains unknown whether psychopathic traits are associated with abnormal N2pc amplitude for targets (i.e., whether the exaggerated attention set revealed by contingent capture to probes translates to an advantage in the deployment of spatial attention to actual targets) or distractors presented at the same time as targets. Additionally, research into lateralized ERP components that complement the N2pc has demonstrated that the human visual system restricts focus to relevant stimuli not only by selecting targets, but also by suppressing the location of salient distractors.

ERP researchers are able to isolate distractor specific activity by presenting targets on the vertical midline of displays and distractors at lateral locations. The distractor positivity $\left(\mathrm{P}_{\mathrm{D}}\right)$ occurs during a similar time window as the N2pc but consists of a positive deflection at contralateral scalp relative to ipsilateral scalp (Hickey, Di Lollo, \& McDonald, 2009). The component indexes a spatial filtering mechanism that complements target selection by suppressing competing distractors (Gaspar \& McDonald, 2014; Luck \& Kappenman, 2013). When a search display contains a salient distractor, faster responses are characterized by an increase in $\mathrm{P}_{\mathrm{D}}$ amplitude compared with slower responses (Gaspar \& McDonald, 2014; McDonald et al., 2013; Sawaki, Geng, \& Luck, 2012). This indicates that $\mathrm{P}_{\mathrm{D}}$ amplitude reflects a compensatory mechanism that prevents distracting stimuli from interfering with (i.e., slowing) target selection. Between conditions/tasks, relative increases in $\mathrm{P}_{\mathrm{D}}$ amplitude or latency indicate the degree to which the task demands visual spatial suppression (Burra, Barras, Coll, \& Kerzel, 2016; Burra \& Kerzel, 2014; Gaspar, Christie, Prime, Jolicœur, \& McDonald, 2016). However, between participants, relative increases in $\mathrm{P}_{\mathrm{D}}$ amplitude or latency capture individual differences in the degree to which visual spatial suppression is employed as an attention control strategy. Failure to incorporate peripheral material may reflect hyper-suppression of task-irrelevant information. If this is the case, then PPI-R scores would be expected to correlate positively with amplitude of the $\mathrm{P}_{\mathrm{D}}$ component evoked by a search display containing both a target and a salient distractor.

Once selected, if an item is actively retained in visual shortterm memory, an N2pc may be followed by a contralateral delay activity (CDA) component, observed approximately $400 \mathrm{~ms}$ after stimulus onset as a sustained negative voltage deflection at the same posterior contralateral versus ipsilateral scalp locations as the N2pc (Jannati et al., 2013; Jolicœur, Brisson, \& Robitaille, 2008; Vogel \& Machizawa, 2004). The negative amplitude CDA is associated with stimulus maintenance and manipulation in working memory. It was first observed by Vogel and Machizawa (2004) in a study of visual working memory during the retention intervals that occurred between presentations of memory and test arrays. In that study, the component was correlated with memory load, as well as individual differences in visual working memory capacity for simple stimuli (shapes). Further research has found that CDA amplitude also indexes the load and capacity of maintaining representations of faces in working memory (Towler, Kelly, \& Eimer, 2015). More recently, evidence for its involvement in working memory manipulation comes from studies observing significant negative voltage CDA amplitude in compound search tasks - paradigms in which a target must be evaluated on a dimension unrelated to the search following its detection (Gaspar \& McDonald, 2014; Jannati, Gaspar, \& McDonald, 2013; Mazza, Turatto, Umiltà, \& Eimer, 2007).

Intriguingly, in a recent ERP investigation of working memory encoding, lateralized distractors elicited a positive amplitude CDA at contralateral scalp locations from 350$750 \mathrm{~ms}$ after stimulus onset in a series of three separate experimental replications (Feldmann-Wüstefeld \& Vogel, 2018). The strength of this positive CDA was positively correlated with the number of distractors present, as well as with individual differences in visual working memory capacity. The authors of the study proposed that an "inverted" or positive amplitude CDA to distractors may reflect sustained or working memory suppression of these items, possibly by tagging distractor locations as "irrelevant" (Feldmann-Wüstefeld \& Vogel, 2018). In line with this interpretation, existing research on cued recall has found that when participants recall a previously viewed search display that contained a lateralized singleton, a negative amplitude CDA-like component is observed if the singleton was a target, but a positive amplitude CDAlike component is seen instead if the singleton was a distractor (Fortier-Gauthier, Moffat, Dell'Acqua, McDonald, \& Jolicœur, 2012). Thus, the visual working memory processes indexed by the CDA likely preserve, reactivate, or directly employ the same retinotopically based mechanisms of the $\mathrm{N} 2 \mathrm{pc}$ and $\mathrm{P}_{\mathrm{D}}$. As such, a significant correlation of PPI-R scores with negative amplitude CDA to targets would therefore indicate their hyper-selection, whereas a significant correlation with positive amplitude CDA to distractors would indicate their hyper-suppression. However in either case, a CDA effect would support the interpretation that the attention bottleneck associated with psychopathic personality traits extends into a later working memory domain of visual-spatial processing.

These selection and suppression mechanisms are guided towards specific items by salience, a property computed by visual cortical areas as a function of an item's distinctiveness on key physical feature-dimensions, such as shape and color (Wolfe, 2007; Wolfe \& Horowitz, 2004). This salience mapping is reflected in a positive amplitude ERP component that 
is greater at posterior contralateral versus ipsilateral sites approximately $100 \mathrm{~ms}$ following stimulus presentation, a difference referred to as the Positivity, posterior contralateral (Ppc; Fortier-Gauthier et al., 2012; Jannati et al., 2013). During this early sensory stage, salient singletons elicit a Ppc regardless of whether they are targets or distractors (Luck \& Hillyard, 1994a). Greater Ppc amplitude has been observed to singletons that occur at cued spatial locations (Hopfinger \& Ries, 2005; Livingstone, Christie, Wright, \& McDonald, 2017), an effect that is likely driven by visual-cortex mechanisms similar - if not identical - to those underlying the wellestablished finding of enhanced P1 responses to spatially cued targets and probes (Vogel \& Luck, 2000). However, greater Ppc amplitude also has been observed to spatially variant target color singletons presented within an array composed entirely of color singletons, supporting the conclusion that top-down enhancement of a singleton's task-relevant features modulates the component's strength even in the absence of spatial cues (Christie, Livingstone, \& McDonald, 2015). Thus, the component captures the cumulative bottom-up and top-down strength of a singleton's "attend-to-me signal" that guides subsequent spatial-attention selection if that item is a target (which would be indexed by an N2pc) or suppression if that item is a distractor (which would be indexed by a $\mathrm{P}_{\mathrm{D}}$ ). A correlation of Ppc strength with PPI-R scores could therefore implicate one of many different attention mechanisms, depending on whether the eliciting singleton is a distractor or target, and whether the component amplitude mediates the relationship between the PPI-R and any of the other visual spatial attention ERP components described above.

To examine the impact of psychopathic personality traits on these ERP components, two tasks with identical stimuli but differing response requirements were employed with an undergraduate sample. In the first, search for a salient singleton evoked the target Ppc, N2pc, and CDA components. A positive association between PPI-R score and the strengths ${ }^{2}$ of these components would suggest that the attention bottleneck involves hyper-selection of target features, spatial location, or working memory representation. In the second task, the singleton was a salient distractor designed to elicit the Ppc, $\mathrm{P}_{\mathrm{D}}$, and CDA components. If PPI-R score was positively associated with the $\mathrm{P}_{\mathrm{D}}$ or positive CDA components, or negatively associated with strength of the Ppc component, this would

\footnotetext{
${ }^{2}$ ERP component "strength" as used here is essentially a proxy term for the component's absolute amplitude value (i.e., value in $\mathrm{mV}$ or $\mu \mathrm{V}$ irrespective of sign). Large positive values for $\mathrm{Ppc}, \mathrm{P}_{\mathrm{D}}$, and positive CDA amplitudes reflect strong responses, and large negative values for N2pc and negative CDA amplitudes reflect strong responses. Statistically, a positive association with a positive amplitude component is indicated by a positive correlation, but a positive association with a negative amplitude component is indicated by a negative correlation. Note that component polarity alone rarely indicates a particular underlying cellular action. See Luck $(2014$, p. 42) for a discussion of how cellular mechanisms, cortical structure, and recording methodologies determine component polarity at the scalp.
}

suggest that the bottleneck instead arises from hypersuppression of distractor spatial location or features respectively.

\section{Methods}

Before data collection, this study was reviewed by the Simon Fraser University Office of Research Ethics and received a minimal risk designation.

\section{Participants and procedure}

A sample of 80 undergraduate students was recruited from the Simon Fraser University Psychology research participation pool (age $M(s)=21.08$ (3.60); 64 women and 16 men, 3 left-handed, 48 English first-language speakers, all with normal color vision as confirmed with Ishihara Color Test number plates). This sample size was chosen to ensure adequate power $(0.80)$ to detect effects of medium size in $F$ tests incorporating three or less independent variables with $\alpha=0.05$, following attrition (Cohen, 1992). After providing informed consent, participants completed a demographic and medical history questionnaire, and the PPI-R self-report measure (described below). EEG was then recorded while participants performed the two visual search tasks in a counterbalanced order. Sessions lasted approximately 2 hours, and participants received course credit for their time.

\section{Psychopathic Personality Inventory-Revised (PPI-R; Lilienfeld \& Widows, 2005)}

Psychopathic personality traits were measured using the Psychopathic Personality Inventory-Revised (PPI-R; Lilienfeld \& Widows, 2005) - a 154-item self-report designed to assess such traits across broad, normative samples. Participants respond to items on the questionnaire by indicating whether a particular statement is False, Mostly False, Mostly True, or True, with these anchors corresponding to a 4-point Likert-type scale. The measure yields a total score and scores on two higher-order factors: Fearless Dominance (FD), which captures emotional and interpersonal aspects of psychopathic personality, and Self-Centered Impulsivity (SCI), which captures impulsive traits and irresponsible lifestyle (Benning et al., 2003).

Consistent with scoring guidelines, total and factor scores were computed as unit-weighted composites. Scores were treated as continuous interval variables for all statistical analyses; however, purely for graphical depictions, tertial scores were used to separate participants into low- $\left(\leq 33^{\text {rd }}\right)$, medium$\left(34^{\text {th }}\right.$ to $\left.66^{\text {th }}\right)$, and high-trait $\left(\geq 67^{\text {th }}\right)$ groups. 


\section{Cognitive Tasks}

Two tasks were adapted from a paradigm that has been employed extensively in the visual search literature to test visual-spatial attention and elicit lateralized ERPs, including the Ppc, N2pc, $\mathrm{P}_{\mathrm{D}}$, and CDA (Christie et al., 2015; Gaspar et al., 2016; Gaspar \& McDonald, 2014; Hickey et al., 2009; Jannati et al., 2013; McDonald et al., 2013; Theeuwes, 2010). Both tasks were programmed and run in E-Prime 2.0.8 on a Windows PC. Participants were seated with their eyes $60 \mathrm{~cm}$ from a 19-inch LCD monitor (1,024 x 786 resolution, $60-\mathrm{Hz}$ refresh rate). Responses were recorded using a Logitech gamepad.

Stimuli were presented on a black background, and consisted of a circular array of eight bright green unfilledshapes $($ red $=0$, green $=255$, blue $=0)$ distributed evenly around a central light grey (E-Prime color attribute setting = "silver") fixation cross (Figure 1). Within the arrays, seven filler shapes were circles (4-cm diameter), whereas one salient singleton was a diamond $\left(4.5 \mathrm{~cm}^{2}\right)$. A $0.2-\mathrm{cm} \times 2.5-\mathrm{cm}$ silver bar was centered within each shape and was oriented horizontally or vertical at random with equal probability on every trial. Shapes were placed with their centers $10 \mathrm{~cm}$ from the center of the screen, at angles of $22.5^{\circ}, 67.5^{\circ}, 112.5^{\circ}, 157.5^{\circ}$, $202.5^{\circ}, 247.5^{\circ}, 292.5^{\circ}$, and $337.5^{\circ}$, leaving $5.8 \mathrm{~cm}$ between the center points of neighbors (Figure 1). On every trial, the salient diamond singleton appeared at one of the eight possible locations (determined at random with equal probability for all locations, and the fixation cross luminance became brighter ("white") or darker ("grey")).
For the visual search task (Task A), participants were instructed to identify the orientation of the bar within the salient diamond singleton as quickly and accurately as possible without moving their eyes from the fixation cross. This was a compound search, because it required participants to first locate the target singleton based on its salient dimension (shape), and then evaluate it on a new dimension that was unrelated to the search (bar orientation). For the fixation task (Task B), participants identified whether the fixation cross had become brighter or darker and ignored the surrounding array, making the singleton a salient distractor. Stimulus arrays offset $100 \mathrm{~ms}$ after response and were followed by a jittered interstimulus interval ( 800 to $1,300 \mathrm{~ms}$ ) before the start of the next trial. Participants performed 18 blocks of 30 trials for each task, with 15 -second breaks between blocks. Trials were rejected from behavioral analyses if participant reaction time (RT) was excessively fast or slow $(150 \mathrm{~ms}<\mathrm{RT}<2,500$ $\mathrm{ms})$.

\section{EEG recording and processing}

EEG activity was recorded using an electrode cap with sintered $\mathrm{Ag} / \mathrm{AgCl}$ active electrodes placed at 64 standard 1010 locations (Biosemi Active Two amplifier, Amsterdam). Additional bilateral active electrode pairs were placed over mastoids, external canthi (to detect horizontal eye movements), and infraorbital locations (to detect vertical eye movements and blinks). Voltages were recorded against a common

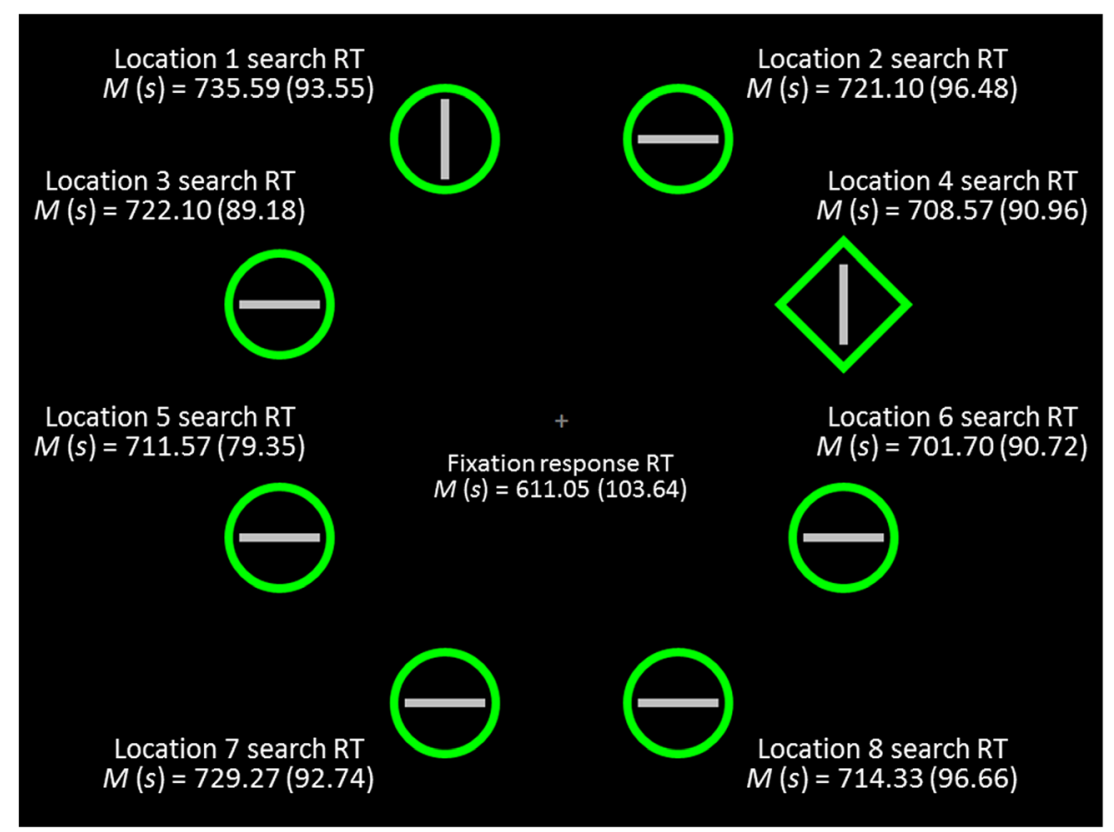

Fig. 1 Sample stimulus display with grand average reaction times (RTs) for the two tasks. Task A (visual search) RTs provided at each potential singleton location; Task B (fixation target, peripheral distractor inhibition) RT across all singleton locations provided at the fixation point. During
ERP analyses, trials were collapsed across bar orientations within tasks and singleton visual hemifields. Manipulation checks confirmed that the differences between various target location in the visual search task were negligible 
mode sense (CMS) active electrode. All data were recorded at a sampling rate of $512 \mathrm{~Hz}$.

Offline EEG data processing was conducted in MATLAB (R2016a) using the Field Trip Toolbox (version 2016.05.10; Oostenveld, Fries, Maris, \& Schoffelen, 2011). For each participant, segments of EEG time-locked to stimulus onset of correct response trials were sampled from continuous EEG, demeaned across the entire epoch, and re-referenced to average mastoids. Independent component analysis (ICA) decomposed the data to 20 unique spatial-temporal signals, from which up to 4 representing ocular artifacts (vertical and lateral saccades, blink propagation, or electromyographic activity) were identified and removed (Jung et al., 1998a; 1998b). Recomposed data was digitally high-pass filtered $\left(0.05 \mathrm{~Hz}, 4^{\text {th }}\right.$ order, Butterworth) and baselined to a period from -200 to $0 \mathrm{~ms}$ before the stimulus onset. Trials remaining contaminated by blinks, saccades, or skeletal movement were rejected based on visual inspection of data segments identified using a semi-automatic artifact detection procedure that assessed electrode z-normalized voltages for extreme values.

Time-locked ERP waveforms were computed for each participant for each task, collapsed across fixation-cross luminance and singleton-bar orientation for the various array configurations. Previous research has found that $\mathrm{P}_{\mathrm{D}}$ component amplitude is greatest for faster response speeds (Gaspar et al., 2016). As such within each task, fast-response and slowresponse waveforms were computed based on whether the participant's trial RT was less than or greater than their median RT for the task. For three pairs of bilateral occipito-parietal electrode sites at which the ERP component effects were measured and/or visualized $(\mathrm{O} 1 / \mathrm{O} 2, \mathrm{PO} 7 / \mathrm{PO} 8$, and $\mathrm{P} 7 / \mathrm{P} 8)$, ipsilateral-electrode waveforms and contralateral-electrode waveforms were computed by collapsing left or right electrodes across trials based on singleton visual hemifield. ${ }^{3}$ Contralateral-minus-ipsilateral ERP difference waveforms were also computed to isolate singleton-related activity. Participants with fewer than 30 artifact-free trials for a given task were rejected from statistical analyses, however the average number of artifact-free trials per task for the participants who were retained far exceeded this threshold (see Participant exclusions and attrition below). Finally, before export for statistical analyses or visualizations, participant averages were digitally low-pass filtered with a $28-\mathrm{Hz}$ cutoff for mean amplitude values or a $10-\mathrm{Hz}$ cutoff for latency values (both $4^{\text {th }}$ order Butterworth). These specific, differing low-pass filter settings for amplitude versus latency were chosen to optimize data quality and limit data distortion within each type of

\footnotetext{
$\overline{3}$ For example, the $\mathrm{O} 1 / \mathrm{O} 2_{\text {contralateral waveform was created by pooling channel }}$ O1 (left occipital scalp) for all trials with a right-lateralized singleton and channel O2 (right occipital scalp) for all trials with a left-lateralized singleton, whereas the $\mathrm{O} 1 / \mathrm{O} 2_{\text {ipsilateral }}$ waveform was created by pooling channel $\mathrm{O} 1$ for all trials with a left-lateralized singleton and $\mathrm{O} 2$ for all channels with a right lateralized singleton.
}

analysis, based on recommendations by Luck (2014, pp. 245-246, p. 300).

\section{Data analysis}

\section{Behavioral Performance.}

Difficulty was compared between the two tasks using paired samples $t$-tests on mean accuracy and RT (within-subjects comparisons of search versus fixation performance). Impact of psychopathic traits on behavior was assessed by examining the correlation of PPI-R total and factor scores with mean accuracy and correct response median RT for each task.

\section{ERP amplitude effects.}

For both tasks, strengths of the Ppc, N2pc, $\mathrm{P}_{\mathrm{D}}$, and CDA components were measured by extracting mean amplitudes for bilateral electrode pairs (contralateral and ipsilateral to the singleton) selected on the basis of previous literature. The time window of the Ppc was centered on the P1 peak of the grand average contralateral waveform, whereas windows for the $\mathrm{N} 2 \mathrm{pc}, \mathrm{P}_{\mathrm{D}}$, and CDA were centered on appropriate peaks or troughs of grand average contralateral-minusipsilateral difference waveforms. The Ppc was tested at occipital sites $\mathrm{O} 1 / \mathrm{O} 2$ from 75 to $125 \mathrm{~ms}$ (Jannati et al., 2013). The $\mathrm{N} 2 \mathrm{pc}$ and $\mathrm{P}_{\mathrm{D}}$ were tested at sites PO7/PO8 from 250 to $310 \mathrm{~ms}$ (Gaspar et al., 2016; Gaspar \& McDonald, 2014; Luck, 2014). Negative and positive contralateral-minus-ipsilateral mean amplitude differences were taken as evidence of the N2pc and $\mathrm{P}_{\mathrm{D}}$ respectively. The CDA was tested at sites $\mathrm{PO} 7 / \mathrm{PO}^{4}$ over two time windows, 350 to $410 \mathrm{~ms}$ and 450 to $510 \mathrm{~ms}$ (Gaspar \& McDonald, 2014; Jannati et al., 2013; Jolicœur et al., 2008; Mazza et al., 2007; Vogel \& Machizawa, 2004).

Significance of the Ppc and $\mathrm{N} 2 \mathrm{pc} / \mathrm{P}_{\mathrm{D}}$ within each task was first confirmed across the entire sample with rANOVAs comparing contralateral versus ipsilateral electrode mean amplitudes as a two-level within subjects-factor of hemisphere, and fast- versus slow-response trials as a two-level within subjects factor of speed. The rANOVAs on the CDA within each task included hemisphere and speed, and a third two-level withinsubjects factor of epoch (mean amplitude measured in the early or late window). All rANOVAs were followed by paired samples $t$-tests (Bonferroni corrected) to assess significant interactions. Further analyses tested whether components were moderated by psychopathic personality by incorporating PPI$\mathrm{R}$ total or factor scores as covariates in repeated measures

\footnotetext{
${ }^{4}$ Note that under the 64-channel 10-10 system electrode locations PO7 and PO8 are roughly analogous to non-standard occipital-left and occipital-right (OL/OR) locations added to the 16-channel 10-20 system and used for the original analysis of the CDA by Vogel \& Machizawa (2004).
} 
analyses of covariance (rANCOVAs) using the same withinsubjects factor(s), though no specific predictions were made regarding the contribution of factor scores as moderators. For rANCOVAs revealing significant moderation of a component by a PPI-R score, contralateral-minus-ipsilateral electrode mean amplitude differences for that component were correlated with that measure to clarify the direction and strength of the relationship (as outlined by Barron \& Kenny, 1986, and Cohen, Cohen, West \& Aiken, 2003).

\section{ERP latency effects}

Latency of the N2pc was extracted for Task A given that the component was anticipated to play a role in visual search. $\mathrm{N} 2 \mathrm{pc}$ latency was defined as the $50 \%$ fractional negative area between a 175 to $400 \mathrm{~ms}$ post-stimulus interval for the PO7/ PO8 contralateral-minus-ipsilateral difference wave (averaged across fast- and slow-response subsets) - that is, the time point by which $50 \%$ of the signed negative area under the curve fell (as recommended by Luck, 2014). In contrast, latency of the $\mathrm{P}_{\mathrm{D}}$ was extracted for Task $\mathrm{B}$, and defined as the $50 \%$ fractional positive area at the same electrodes and between the same post-stimulus interval.

Participant component latencies were correlated with median RT and accuracy to assess the impact of target localization on search efficiency in the case of the N2pc, and distractor suppression on task performance in the case of the $\mathrm{P}_{\mathrm{D}}$. Moderation of these associations by psychopathic traits was tested through multiple linear regressions incorporating PPI-R total or factor scores and interaction terms (as described by Baron \& Kenny, 1986; Cohen et al., 2003).

\section{Results}

\section{Participant exclusions and attrition}

Three participants were unable to perform Task A due to time constraints during their recording sessions. One participant was unable to completed Task B due to time constraints. Task A data for two participants were excluded due to poor ocular EEG quality, and for a third due to a software issue that resulted in their responses being lost. Task B data for four participants were excluded due to overall EEG quality. These exclusions resulted in a final sample of 69 participants (age $M(s)=20.75$ (2.637); 56 women and 13 men, 3 lefthanded, 43 English first-language speakers; PPI-R total $M(s)$ $=273.5$ (26.9); SCI $M(s)=137.0$ (16.9); FD $M(s)=106.4$ (17.9)). PPI-R total, factor, and subscale means, variances, and covariances for these 69 participants are provided in Table 1. Following rejection of trials for inaccurate responses and ocular artifact contamination, participants ERP averages included an average of 481.8 trials $(s=81.10)$ or $91.9 \%$ of trials across conditions for task A, and an average of 489.5 trials $(s=67.25)$ or $92.1 \%$ of trials across conditions for task B.

\section{Behavioral data and between task performance}

Analysis of behavioral data between tasks indicated that both were performed with a high degree of accuracy (Task A: $M(s)$ $=0.961(0.0352)$; Task B: $M(s)=0.966(.0347) ; t_{\text {task } A-}$ taskB $(68)=-1.019, p=0.312)$. However, slower median RT in Task A $(M(s)=715.1 \mathrm{~ms}(82.7))$ than Task B $(M(s)=$ $611.1 \mathrm{~ms}$ (103.6)) confirmed that an initial search was required before participants could respond to peripheral targets $\left(t_{\text {taskA-taskB }}(69)=11.555, p<0.001,95 \% \mathrm{CI}_{\text {difference }}[86.1\right.$, 120.0]). PPI-R total and factor scores were not associated with accuracy or correct response RT for either task (Table 2).

\section{Task A visual search ERPs}

Mean amplitude analyses confirmed that target singletons evoked typical Ppc, N2pc, and CDA components across the full sample in the visual search task (Table 3; Figure 2.A). That is to say, the task functioned normally, and elicited the desired ERP effects.

First, during the time window of the Ppc, contralateral hemisphere voltage was significantly more positive than ipsilateral hemisphere voltage $\left(0.126 \mu \mathrm{V} ; F_{\text {hemisphere }}(1,68)=\right.$ 22.394, $p<0.001, \eta_{\mathrm{p}}{ }^{2}=0.248,95 \%$ CI [0.073, 0.180]). This demonstrated that participants did indeed find the target singletons visually salient. There was no significant main effect or interaction of speed with Ppc amplitude (for both, $\left.F(1,68) \leq 1.116, p \geq 0.294, \eta_{\mathrm{p}}^{2} \leq 0.016\right)$, meaning that this salience did not vary between fast- and slow-response trials.

Second, the rANOVA on amplitude during the N2pc time window returned a significant main effect of hemisphere $\left(F_{\text {hemisphere }}(1,68)=94.572, p<0.001, \eta_{\mathrm{p}}^{2}=0.582\right)$, and a significant interaction of hemisphere with speed $\left(F_{\text {hemisphere }} *_{\text {speed }}(1,68)=6.207, p=0.015, \eta_{\mathrm{p}}{ }^{2}=0.084\right)$. Follow-up tests confirmed the presence of an N2pc component, with contralateral hemisphere voltage significantly more negative than ipsilateral hemisphere voltage for both fastresponse trials $(-1.305 \mu \mathrm{V} ; t(68)=-8.385, p<0.001,95 \%$ CI $[-1.616,-0.995])$ and slow-response trials $(-0.992 \mu \mathrm{V}$; $t(68)=-9.214, p<0.001,95 \%$ CI $[-1.206,-0.777])$. This effect was significantly stronger for fast-response than slowresponse trials $(-0.314 \mu \mathrm{V} ; t(68)=-2.491, p=0.015,95 \% \mathrm{CI}$ $[-0.565,-0.062])$, indicating that efficient target selection at the physiological level corresponded to more efficient behavioural responses.

Third, the rANOVA assessing the CDA returned significant main effects and interactions for hemisphere, speed, and epoch $\left(F_{\text {hemisphere }}(1,68)=9.046, p=0.004, \eta_{\mathrm{p}}{ }^{2}=0.117\right.$; $F_{\text {epoch }}(1,68)=19.515, p<0.001, \eta_{\mathrm{p}}{ }^{2}=0.223 ; F_{\text {speed }}(1,68)=$ 
Table 1 Psychopathic personality inventory-revised mean, variance, and covariance matrix

\begin{tabular}{|c|c|c|c|c|c|c|c|c|c|c|c|c|}
\hline & & 1. & 2. & 3. & 4. & 5. & 6. & 7. & 8. & 9. & 10. & 11. \\
\hline \multirow[t]{2}{*}{ 1. Total score } & $M$ & 273.55 & & & & & & & & & & \\
\hline & $s^{2}$ & 721.00 & & & & & & & & & & \\
\hline \multirow[t]{2}{*}{ 2. Self-Centered Impulsivity } & $r$ & $.700^{* *}$ & 136.98 & & & & & & & & & \\
\hline & $\operatorname{cov}$ & 317.73 & 285.73 & & & & & & & & & \\
\hline \multirow[t]{2}{*}{ 3. Fearless Dominance } & $r$ & $.713^{* *}$ & .067 & 106.40 & & & & & & & & \\
\hline & $\operatorname{cov}$ & 341.76 & 20.16 & 318.79 & & & & & & & & \\
\hline \multirow[t]{2}{*}{ 4. Coldheartedness } & $r$ & $.335^{* *}$ & .102 & .023 & 46.85 & & & & & & & \\
\hline & $\operatorname{cov}$ & 61.50 & 11.84 & 2.81 & 6.85 & & & & & & & \\
\hline \multirow[t]{2}{*}{ 5. Machiavellian Egocentricity } & $r$ & $.639^{* *}$ & $.762^{* *}$ & .100 & $.361^{* *}$ & 41.49 & & & & & & \\
\hline & $\operatorname{cov}$ & 123.79 & 93.01 & 12.93 & 17.85 & 52.08 & & & & & & \\
\hline \multirow[t]{2}{*}{ 6. Rebellious Nonconformity } & $r$ & $.466^{* *}$ & $.533^{* *}$ & $.249^{*}$ & -.134 & .219 & 32.53 & & & & & \\
\hline & $\operatorname{cov}$ & 74.91 & 53.87 & 26.54 & -5.50 & 9.43 & 35.77 & & & & & \\
\hline \multirow[t]{2}{*}{ 7. Carefree Nonplanfulness } & $r$ & $.336^{* *}$ & $.620^{* *}$ & -.111 & .075 & $.362^{* *}$ & .078 & 33.93 & & & & \\
\hline & $\operatorname{cov}$ & 57.04 & 66.29 & -12.49 & 3.24 & 16.51 & 2.97 & 39.98 & & & & \\
\hline \multirow[t]{2}{*}{ 8. Blame Externalization } & $r$ & $.344^{* *}$ & $.640^{* *}$ & -.057 & -.082 & $.310^{* *}$ & .142 & .161 & 29.02 & & & \\
\hline & $\operatorname{cov}$ & 61.99 & 72.57 & -6.82 & -3.76 & 14.99 & 5.70 & 6.83 & 45.05 & & & \\
\hline \multirow[t]{2}{*}{ 9. Fearlessness } & $r$ & $.649^{* *}$ & .227 & $.770^{* *}$ & -.024 & .163 & $.405^{* *}$ & -.078 & .109 & 32.19 & & \\
\hline & $\operatorname{cov}$ & 155.93 & 34.35 & 123.06 & -1.48 & 10.52 & 21.67 & -4.40 & 6.56 & 80.08 & & \\
\hline \multirow[t]{2}{*}{ 10. Stress Immunity } & $r$ & $.336^{* *}$ & -.211 & $.600^{* *}$ & $.274^{*}$ & -.104 & -.069 & -.116 & $-.248^{*}$ & .237 & 29.56 & \\
\hline & $\operatorname{cov}$ & 59.73 & -23.59 & 70.90 & 12.42 & -4.96 & -2.72 & -4.86 & -11.04 & 14.02 & 43.85 & \\
\hline \multirow[t]{2}{*}{ 11. Social Influence } & $r$ & $.516^{* *}$ & .061 & $.768^{* * *}$ & -.131 & .112 & .139 & -.056 & -.038 & $.356^{* * *}$ & .216 & 44.65 \\
\hline & $\operatorname{cov}$ & 126.10 & 9.40 & 124.83 & -8.13 & 7.37 & 7.59 & -3.22 & -2.33 & 28.97 & 13.04 & 82.83 \\
\hline
\end{tabular}

Note: $N=69$; bolded and italicized values within cells on the diagonal are mean and variance (respectively) for the given scale; cov $=$ covariance; $r=$ Pearson correlation coefficient; $M=$ mean; $s^{2}=$ variance. ${ }^{*} * p \leq 0.01$ (2-tailed). ${ }^{*} p \leq 0.05$ (2-tailed).

$37.760, p<0.001, \eta_{\mathrm{p}}{ }^{2}=0.357 ; F_{\text {hemisphere }}{ }^{\text {epoch }}(1,68)=4.482$, $p=0.038, \eta_{\mathrm{p}}{ }^{2}=0.062 ; F_{\text {hemisphere }}$ speed $(1,68)=5.965, p=$ $0.017, \eta_{\mathrm{p}}{ }^{2}=0.081 ; F_{\text {epoch }}{ }^{\text {speed }}(1,68)=24.126, p<0.001$, $\eta_{\mathrm{p}}{ }^{2}=.262 ; F_{\text {hemisphere }}$ epoch $^{*}$ speed $(1,68)=7.655, p=0.007$, $\left.\eta_{\mathrm{p}}{ }^{2}=0.101\right)$. Follow-up $t$-tests revealed a negative amplitude CDA component during slow-response trials between both the $350-410 \mathrm{~ms}(-0.307 \mu \mathrm{V} ; t(68)=-3.036, p=0.003,95 \% \mathrm{CI}$ $[-0.508,-0.105])$ and $450-510 \mathrm{~ms}$ epochs $(-0.574 \mu \mathrm{V} ; t(68)$ $=-5.234, p<0.001,95 \% \mathrm{CI}[-0.792,-0.354])$, although this effect was significantly greater for the later epoch. Additionally, the CDA contra-minus-ipsi difference was significantly stronger for slow-response trials than fast-response trials $(-0.444 \mu \mathrm{V} ; t(68)=-2.997, p=0.004,95 \%$ CI $[-0.739$, $-0.148])$. Thus, singletons appear to have been maintained in working memory beyond their initial selection, particularly when participants took longer to respond.

\section{N2pc latency during visual search.}

Four participants were excluded from Task A N2pc latency analyses due to the absence of a negative trough in the 175 $400 \mathrm{~ms}$ testing window. Across the remaining 65 participants, mean N2pc latency was $287.8 \mathrm{~ms}(s=21.8)$ following stimulus onset. Participant latency was significantly and positively

Table 2 Psychopathic personality inventory-revised and behavioral performance

\begin{tabular}{llll}
\hline & Total score $r(p)$ & Self-centered Impulsivity $r(p)$ & Fearless Dominance $r(p)$ \\
\hline Task A: visual search $(n=69)$ & & & $0.026(.830)$ \\
Median RT & $-0.017(.887)$ & $-0.027(0.826)$ & $0.193(.113)$ \\
Mean accuracy & $0.184(.130)$ & $0.131(0.285)$ & $0.163(.181)$ \\
Task B: fixation target $(n=69)$ & $0.146(.231)$ & $0.037(0.761)$ & $0.006(.960)$ \\
Median RT & $-0.090(461)$ & $-0.161(0.187)$ & \\
Mean accuracy & & & \\
\hline
\end{tabular}

Note: $r$ = Pearson correlation coefficient; $p=$ statistical test probability; RT = correct response reaction time. No significant correlations were observed. 
Table 3 ERP component amplitudes $(\mu \mathrm{V})$

\begin{tabular}{|c|c|c|c|c|}
\hline Task A: visual search $(n=69)$ & Ppc $75-125 \mathrm{~ms} M(s) *$ & $\mathrm{~N} 2 \mathrm{pc} 250-310 \mathrm{~ms} M(s) * \dagger$ & CDA 350-410 ms $M(s) *{ }^{\dagger \dagger}$ & CDA 450-510 ms $M(s) *^{\dagger+}$ \\
\hline \multicolumn{5}{|l|}{ Fast responses } \\
\hline Contralateral & $1.3487(2.3149)$ & $1.2718(3.5766)$ & $5.0366(3.8068)$ & $4.5507(3.5833)$ \\
\hline Ipsilateral & $1.1960(2.3274)$ & $2.5772(3.7629)$ & $5.1250(3.9429)$ & $4.6804(3.7615)$ \\
\hline Difference & $0.1528(0.3141)$ & $-1.3053(1.2931)$ & $-0.0883(1.0668)$ & $-0.1297(1.1738)$ \\
\hline \multicolumn{5}{|l|}{ Slow responses } \\
\hline Contralateral & $1.3499(2.3448)$ & $1.4554(3.5224)$ & $4.1255(3.5108)$ & $2.8545(3.0638)$ \\
\hline Ipsilateral & $1.2501(2.3603)$ & $2.4471(3.5549)$ & $4.4323(3.5039)$ & $3.4280(3.0130)$ \\
\hline Difference & $0.0998(0.2938)$ & $-0.9917(0.8941)$ & $-0.3068(0.8393)$ & $-0.5736(0.9103)$ \\
\hline Task B: fixation target $(n=69)$ & Ppc $75-125 \mathrm{~ms} M(s) *$ & $\mathrm{P}_{\mathrm{D}} 250-310 \mathrm{~ms} M(s)^{\dagger}$ & CDA $350-410 \mathrm{~ms} M(s) * T^{\bullet}$ & CDA $450-510 \mathrm{~ms} M(s) *^{\dagger \dagger}$ \\
\hline \multicolumn{5}{|l|}{ Fast responses } \\
\hline Contralateral & $0.7899(2.2972)$ & $3.2317(4.6177)$ & $6.2694(4.4987)$ & $4.7458(3.2124)$ \\
\hline Ipsilateral & $0.6831(2.3272)$ & $3.1118(4.5843)$ & $6.1190(4.5224)$ & $4.6255(3.3374)$ \\
\hline Difference & $0.1068(0.2526)$ & $0.1199(0.5851)$ & $0.1504(0.6113)$ & $0.1203(0.7474)$ \\
\hline \multicolumn{5}{|l|}{ Slow responses } \\
\hline Contralateral & $0.6709(2.1474)$ & $2.9121(4.3197)$ & $4.2252(3.7670)$ & $3.9357(3.1723)$ \\
\hline Ipsilateral & $0.5917(2.1108)$ & $2.8898(4.2408)$ & $4.0802(3.7414)$ & $3.7735(3.0154)$ \\
\hline Difference & $0.0792(0.2963)$ & $0.0223(0.6073)$ & $0.1450(0.6400)$ & $0.1622(0.6354)$ \\
\hline
\end{tabular}

Note: Fast-response values were computed for trials with RT $<$ participant median RT, and slow-response trials were computed for trials with RT $\geq$ participant median RT; Ppc values were calculated at contralateral and ipsilateral electrode sites $\mathrm{O} 1 / \mathrm{O} 2 ; \mathrm{N} 2 \mathrm{pc}, \mathrm{P}_{\mathrm{D}}$, and CDA values were calculated at contralateral and ipsilateral electrode sites PO7/PO8; $\mu \mathrm{V}=$ microvolts; $M=$ mean; $s=$ standard deviation. ${ }^{1}$ Difference $=$ contralateral minus ipsilateral electrode site difference; *Significant difference between contralateral versus ipsilateral hemisphere, $p \leq 0.05 ;{ }^{\dagger}$ Significant difference between slowversus fast-response speed, $p \leq 0.05 ;{ }^{*}$ Significant interaction of hemisphere and speed, $p \leq 0.05 ;{ }^{*}$ Significant interaction of Self-Centered Impulsivity factor score with difference between contralateral versus ipsilateral hemisphere, $p \leq 0.05$.

correlated with median RT $(r=0.420, p<0.001)$. Similar to the rANOVA that found stronger N2pc mean amplitude on fast-response trials, this demonstrated a clear relationship between target selection time and behavioral response. N2pc latency and mean accuracy were not significantly correlated $(r=-0.042, p=0.740)$.

\section{No association of PPI-R scores with visual search ERPs.}

Although the search task functioned normally and significant target Ppc, N2pc, and CDA effects were observed in the grand average data, rANCOVA moderation analyses incorporating PPI-R total or factor scores as covariates found no significant interactions of PPI-R measures with any component (for all Ppc interactions, $F(1,67) \leq 1.201, p \geq 0.277, \eta_{\mathrm{p}}{ }^{2} \leq 0.018$; for all N2pc interactions $F(1,67) \leq 2.324, p \geq 0.132, \eta_{\mathrm{p}}{ }^{2} \leq 0.034$; and for all CDA interactions $F(1,67) \leq 1.338, p \geq 0.252, \eta_{\mathrm{p}}{ }^{2} \leq$ 0.020 ; Figures 3 and 4 ). As noted for behavioral performance results above, PPI-R measures were uncorrelated with RT and accuracy. PPI-R scores were also uncorrelated with N2pc latency (for all, $|r| \leq 0.071, p \geq 0.576, n=65$ ). Finally, the association of N2pc latency with RT was not moderated by PPI-R measures, as evidenced by small, non-significant regression coefficients for main-effects and interactions of total and factor scores as predictors of RT (for all, $|b *| \leq b^{*}{ }_{F} *_{\text {latency }}$ $=.211, p=0.070)$. Therefore, on the whole there was no association of psychopathic personality traits with enhanced target salience calculation, visual-spatial selection, or working memory evaluation. This was in spite of good functioning of the visual search task in terms of its ability to evoke typical ERP effects across the full sample.

\section{Task B fixation target, lateral distractor suppression ERPs}

Across the entire sample distractor singletons elicited a significant Ppc component, as indicated by a significant main effect of hemisphere $\left(F_{\text {hemisphere }}(1,68)=18.214, p<0.001, \eta_{\mathrm{p}}{ }^{2}=\right.$ .221). Follow-up tests revealed that this effect was driven by greater positive voltage over the contralateral versus ipsilateral hemisphere, that is, a Ppc component $(+0.093 \mu \mathrm{V} ; t(68)=$ $4.227, p<0.001,95 \%$ CI $[0.050,0.136])$. This showed that although participants were instructed to focus on the fixation point, they still found the singleton distractor salient. As with the results of Task A, absence of a significant main effect or interaction of speed demonstrated that the singleton's perceived salience did not predict whether participants would response quickly or slowly.

Despite the appearance of greater positive mean amplitude at contralateral than ipsilateral electrode sites during the $\mathrm{P}_{\mathrm{D}}$ window of the grand average waveform, this difference failed 


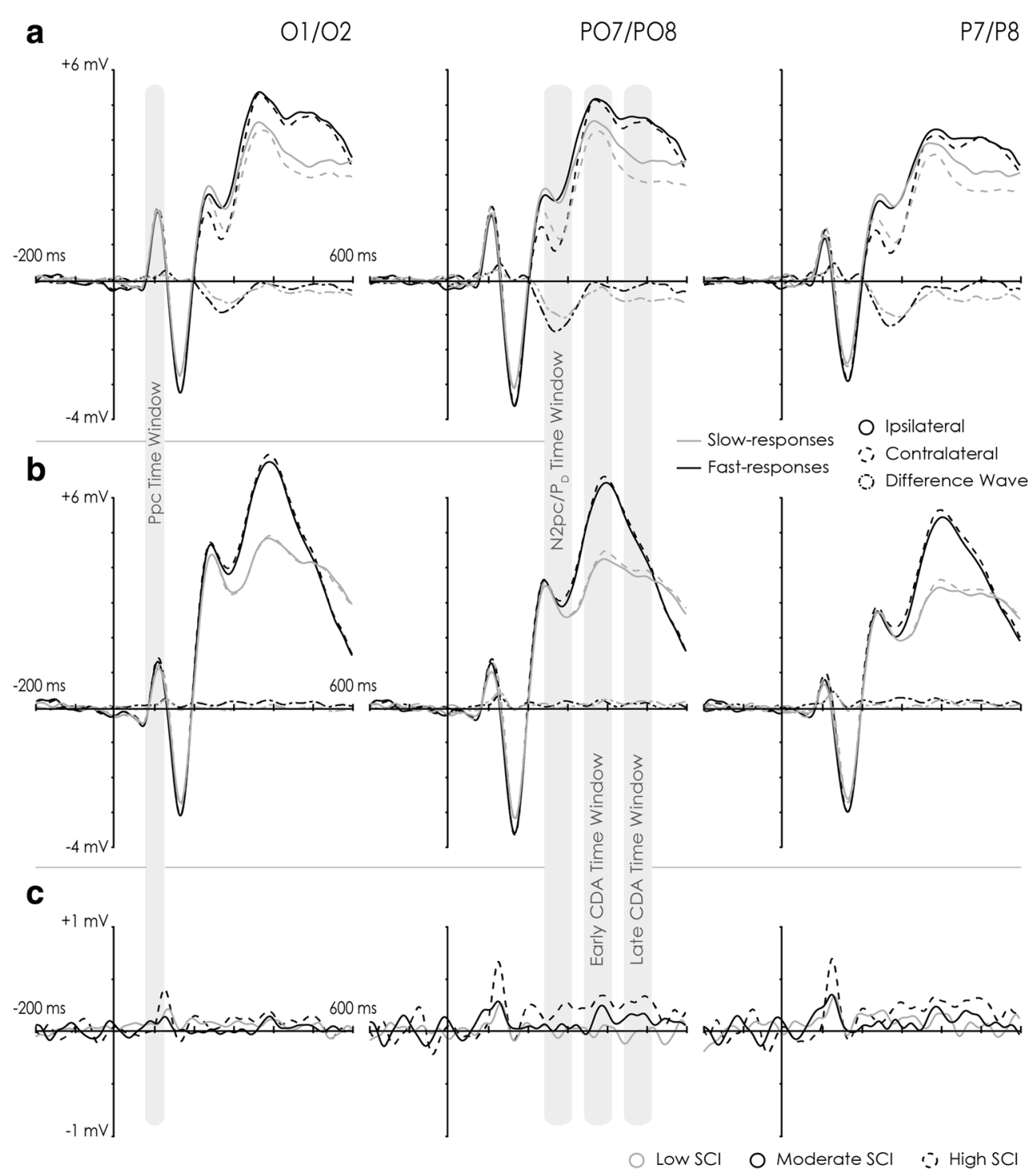

Fig. 2 ERP data $(\mu \mathrm{V})$ at three occipital and parietal electrode sites over the grand average epoch $(-200 \mathrm{~ms}$ to $600 \mathrm{~ms})$. O1/O2, located respectively over left and right occipital scalp, were used in analyses of the Ppc; PO7/PO8, also located over left and right occipital scalp, were used in analyses on the remaining components; $\mathrm{P} 7 / \mathrm{P} 8$, located over left and right parietal scalp, are presented for visualization purposes only. A. Task A visual search grand average ERP waveforms recorded at target ipsilateral (solid-line) and contralateral (dashed-line) hemispheres, and contraminus-ipsi difference (dashed-dotted line). Black waveforms are averaged across fast-response trials, whereas grey waveforms are averaged across slow-response trials (based on a median split of trial reaction times

to reach significance $(+0.071 \mu \mathrm{V}, 95 \%$ CI $[-0.039,0.181$,$] ;$ $\left.F_{\text {hemisphere }}(1,68)=1.662, p=0.202, \eta_{\mathrm{p}}{ }^{2}=0.024\right)$. The main effect and interaction of speed also approached but failed to reach significance, indicating that the $\mathrm{P}_{\mathrm{D}}$ was not associated with efficient responding in the presence of a distractor $\left(F_{\text {speed }}(1,68)=3.652, p=0.060, \eta_{\mathrm{p}}^{2}=0.051\right.$; $\left.F_{\text {hemisphere } *_{\text {speed }}(1,68)}=1.129, p=0.292, \eta_{\mathrm{p}}{ }^{2}=0.016\right)$.

The absence of a significant $\mathrm{P}_{\mathrm{D}}$ component to the singleton distractors was surprising, especially in light of the for each participant). B. Task B fixation target waveforms using the same legend. C. Task B ERP contra-minus-ipsi difference waveforms averaged across fast- and slow-response trials. Data are group by tertiles for Psychopathic Personality Inventory-Revised Self-Centered Impulsivity $(\mathrm{SCI})$ factor low $(M(s)=117.87(10.69) ; \min =93 ; \max =133)$, moderate $(M(s)=138.41(3.27) ; \min =134 ; \max =144)$, and high $(M(s)=154.65$ $(6.93) ; \min =145 ; \max =170)$ scoring participants. Note different legend and y-axis scale. Tertile groupings (each $n=23$ ) are presented only for graphical purposes; all statistical analyses incorporating PPI-R data treated scores as continuous interval variables

aforementioned significant Ppc effect, but tests of the CDA component found evidence of distractor related activity at the working memory evaluation stage. First, a significant effect of hemisphere demonstrated the presence of a CDA component regardless of response speed, and during both windows of the effect $\left(F_{\text {hemisphere }}(1,68)=6.838, p=0.011, \eta_{\mathrm{p}}{ }^{2}=\right.$ 0.091). Follow-up tests revealed that voltage over contralateral scalp was significantly more positive than ipsilateral scalp $(+0.144 \mu \mathrm{V} ; t(68)=2.618, p=0.011,95 \% \mathrm{CI}$ 
Ppc

(75 - $125 \mathrm{~ms} ; \mathrm{Ol} / \mathrm{O} 2)$

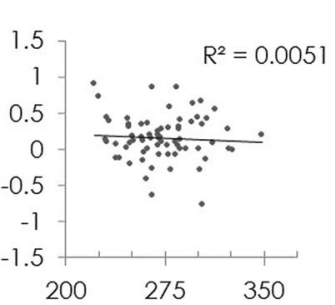

N2pc

(250 - 310 ms; PO7/PO8)
Early CDA

(350 - $410 \mathrm{~ms} ; \mathrm{PO} / \mathrm{PO} 8$ )
Late CDA

(450 - 510 ms; PO7/PO8)
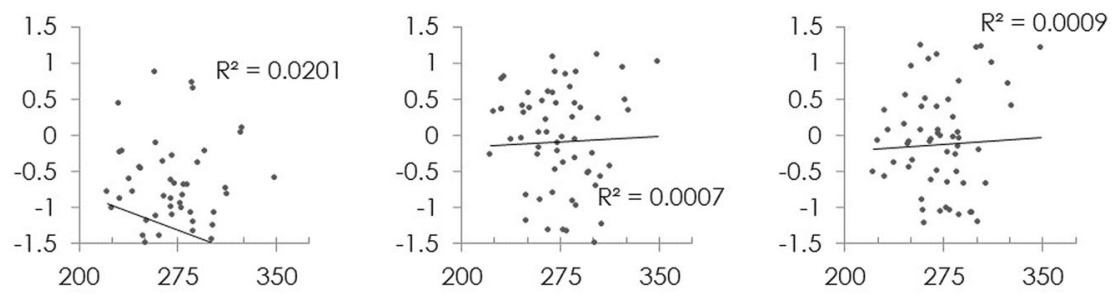

Total Score
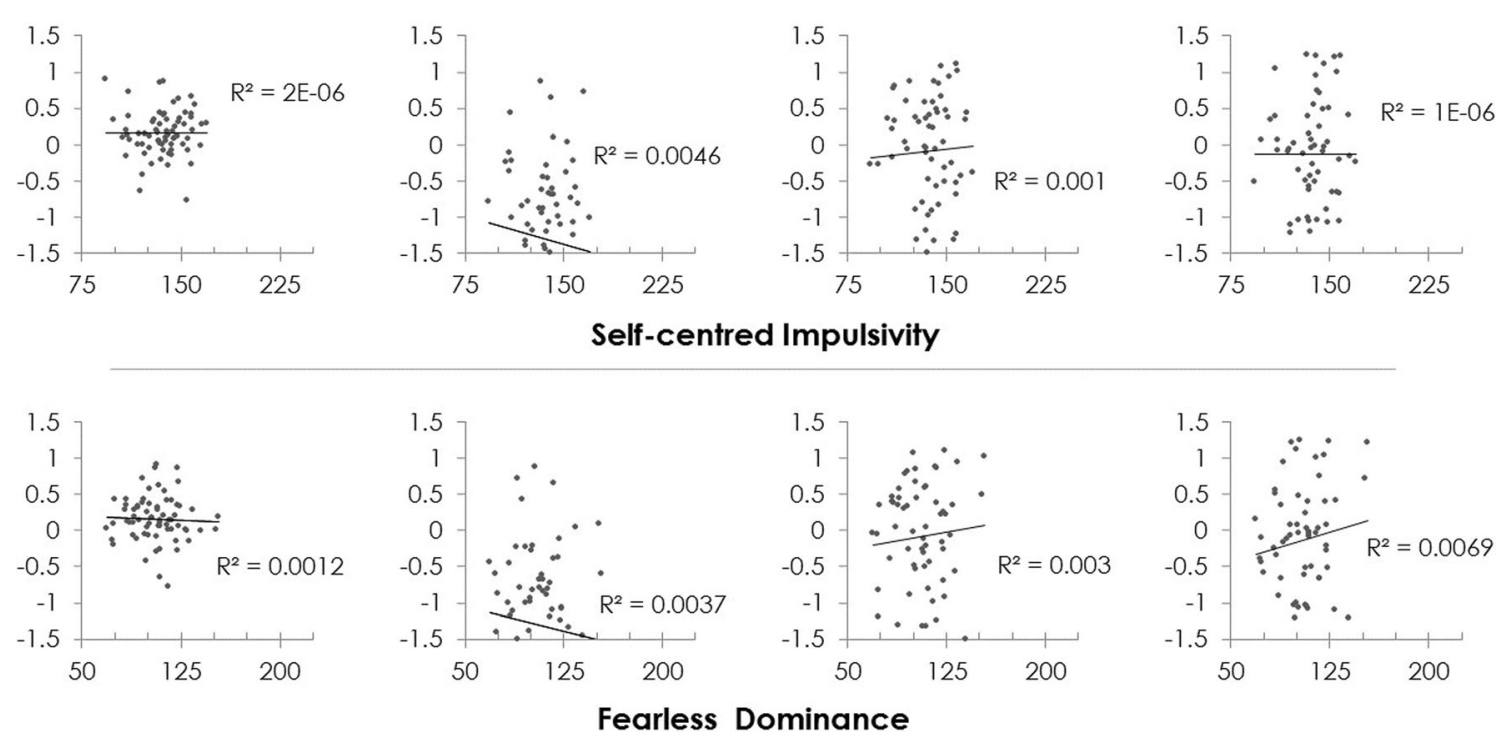

Fearless Dominance

Fig. 3 Visual search task (Task A), fast-response trials. Scatter plots of Psychopathic Personality Inventory-Revised total and factor scores by component grand average contralateral-minus-ipsilateral amplitude differences $(\mu \mathrm{V})$ at electrode sites of interest

$[0.034,0.255])$. This positive amplitude CDA component was surprising given that the $\mathrm{CDA}$ is typically reported to be a negative voltage component observed during working memory retention of target items. However, as discussed previously a positive CDA component elicited by distractors has recently been proposed to reflect a similar suppression mechanism as the $\mathrm{P}_{\mathrm{D}}$ (Feldmann-Wüstefeld \& Vogel, 2018). Significant main effects and a significant interaction of epoch and speed were also observed $\left(F_{\text {epoch }} *_{\text {speed }}(1,68)=24.071, p\right.$ $<0.001, \eta_{\mathrm{p}}{ }^{2}=0.261$ ), revealing that voltage across the entire scalp varied between the two intervals of the CDA, and based on whether participants responded quickly or slowly. However because these effects did not interact with the contralateral versus ipsilateral voltage difference (i.e. the CDA component itself) they were not further explored.

\section{$P_{D}$ latency during fixation response}

Latency of positive voltage activity between $175-400 \mathrm{~ms}$ post-stimulus was examined despite the absence of a significant $P_{D}$ effect across the sample in Task $B$. An adequate positive peak within the time window was present for only 55 participants. Amongst these participants, mean latency of this activity was $290.4 \mathrm{~ms}(s=45.3)$ following stimulus onset. This latency was uncorrelated with RT $(r=-0.035, p=$ 0.802 ), but was significantly and negatively correlated with accuracy $(r=-0.312, p=0.021)$. This negative correlation suggests that participants were most accurate on trials where contralateral versus ipsilateral amplitude differentiation occurred earlier rather than later, which is consistent with previous research on the $\mathrm{P}_{\mathrm{D}}$.

\section{PPI-R SCI scores were associated with distractor related activity.}

Although no relationship was found between PPI-R scores and target selection in Task A, moderation analyses for Task B revealed an impact of Self-Centered Impulsivity (SCI) factor scores on lateralized ERP responses to distractors (see Figure 2.C, in which data is parsed for three groups of participants based on SCI score). For the two components elicited by distractors across the entire sample, the Ppc and the CDA, we ran rANCOVAs incorporating PPI-R factor or total scores as covariates. The rANCOVA of CDA amplitude 
Ppc

(75 - $125 \mathrm{~ms} ; \mathrm{Ol} / \mathrm{O} 2)$

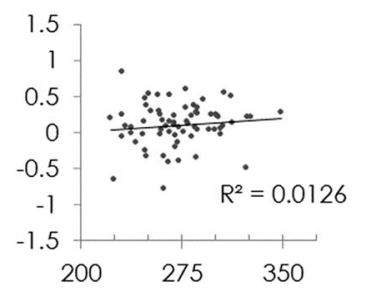

N2pc

(250 - 310 ms; PO7/PO8)

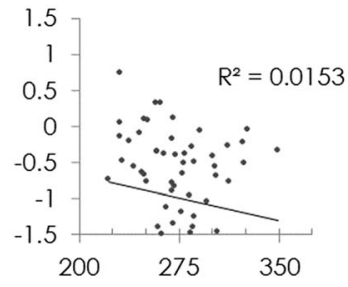

Early CDA

(350 - 410 ms; PO7/PO8)
Late CDA

(450 - 510 ms; PO7/PO8)

Total Score
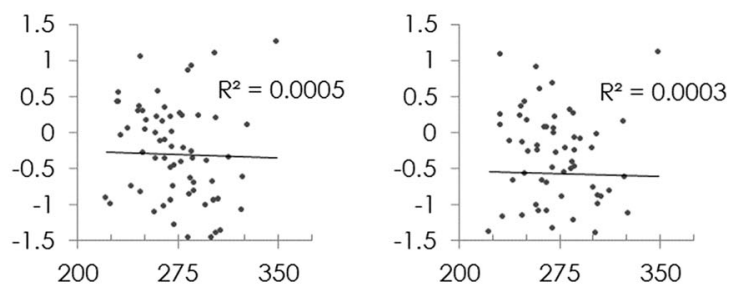

rat
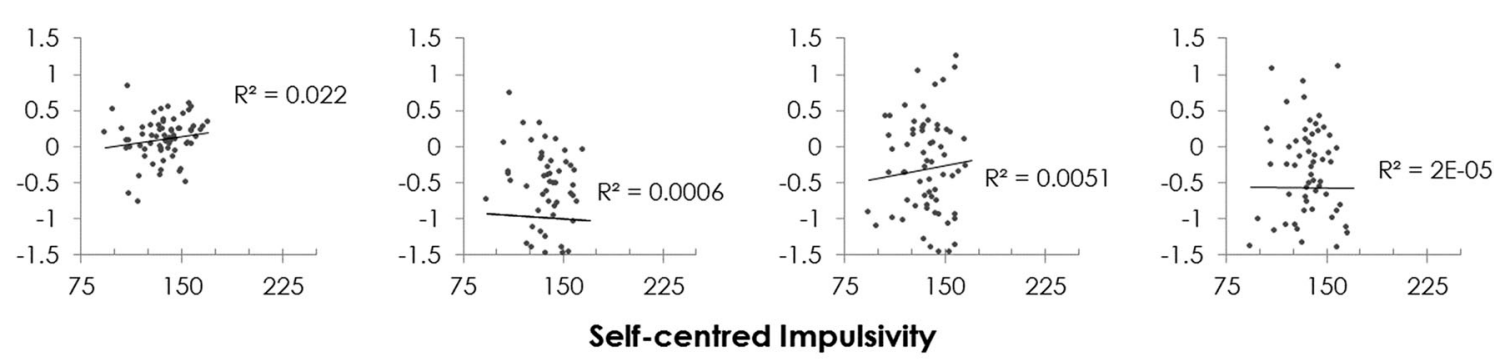

Self-centred Impulsivity
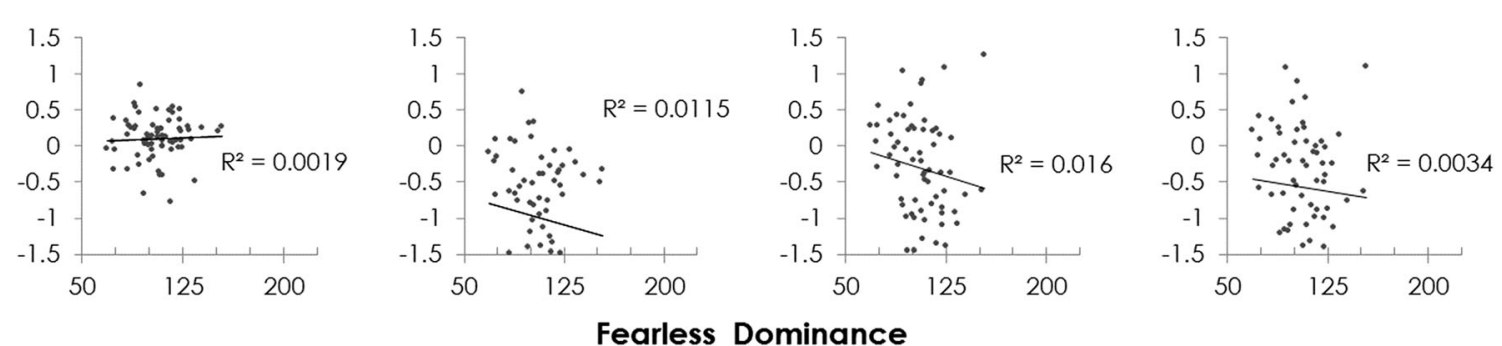

Fearless Dominance

Fig. 4 Visual search task (Task A), slow-response trials. Scatter plots of Psychopathic Personality Inventory-Revised total and factor scores by component grand average contralateral-minus-ipsilateral amplitude differences $(\mu \mathrm{V})$ at electrode sites of interest

with SCI as a covariate produced a significant interaction of hemisphere and SCI $\left(F_{\text {hemisphere }}{ }_{S C I}(1,68)=7.343, p=0.009\right.$, $\eta_{\mathrm{p}}{ }^{2}=0.099$ ). To clarify the direction and strength of this statistical effect, follow-up correlations were run on contralateral-minus-ipsilateral amplitude differences collapsed across the two CDA time windows and two response speeds (as there were no significant interactions observed with the epoch or speed factors). These tests indicated that CDA amplitude became more positive as SCI scores increased ( $r=$ $.314, p=.009$ ). The Ppc was not moderated by SCI (for all interactions, $\left.F(1,68) \leq 1.558, p \geq .216, \eta_{\mathrm{p}}{ }^{2} \leq .023\right)$. PPI-R total score and FD did not moderate either component (for all PPIR total score interactions, $F(1,68) \leq 1.792, p \geq 0.185, \eta_{\mathrm{p}}^{2} \leq$ 0.026 ; for all FD interactions, $F(1,68) \leq 1.651, p \geq 0.203, \eta_{\mathrm{p}}{ }^{2}$ $\leq 0.024$ ).

Thus, the pattern that emerged when accounting for SCI factor scores indicated that individuals highest in these traits drove the significant positive CDA effects observed in the grand averaged data, as seen in the grouped ERP waveforms in Figure 2C and the scatter plots in Figures 5 and 6 . These participants showed more lateralized activity potentially indicative of distractor suppression that lower trait individuals. This finding suggests that the attention bottleneck associated with psychopathic personality traits may be driven by inhibition of distractor information.

\section{Discussion}

Covariance of PPI-R total and factor scores with ERP indices of item salience, selection, suppression, and working memory revealed electrophysiological evidence of abnormal visualspatial attention to distractors but not targets among undergraduates with higher levels of self-centered and impulsive traits (i.e., the SCI factor). This extends previous behavioral and ERP research that has found that inmates with high scores on the PCL-R show resistance to peripherally presented nontarget information (Baskin-Sommers et al., 2012; Hiatt et al., 2004; Newman \& Kosson, 1986; Patterson \& Newman, 1993; Wolf et al., 2012; Zeier et al., 2009). In addition to providing physiological evidence of this behavioral tendency, patterns observed in the current study shed light on the neural mechanism that drives this atypical pattern of attention. The ERP data presented here suggest that these attention abnormalities 
Ppc

(75 - $125 \mathrm{~ms} ; \mathrm{Ol} / \mathrm{O} 2)$

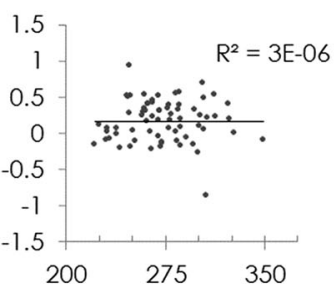

$\mathbf{P}_{\mathrm{D}}$

(250 - $310 \mathrm{~ms} ; \mathrm{PO} / \mathrm{PO} 8)$
Early CDA

(350 - 410 ms; PO7/PO8)
Late CDA

(450 - 510 ms; PO7/PO8)
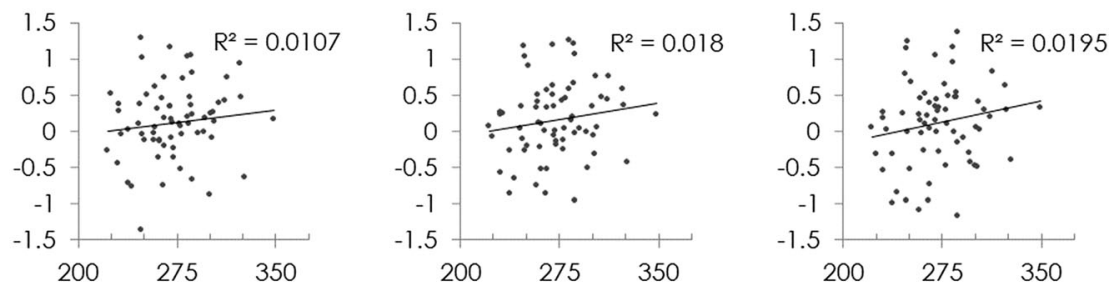

Total Score
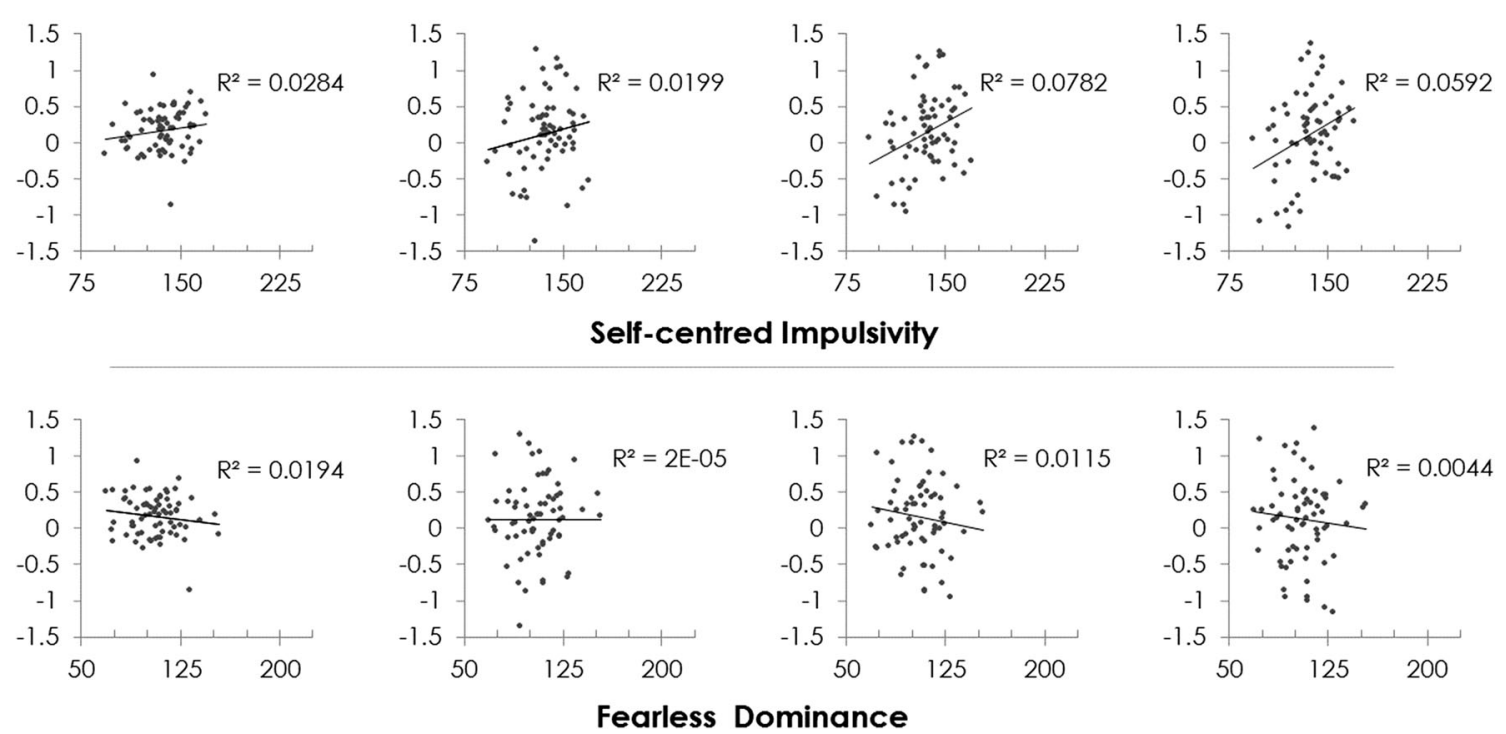

Fearless Dominance

Fig. 5 Fixation task (Task B), fast-response trials. Scatter plots of Psychopathic Personality Inventory-Revised total and factor scores by component grand average contralateral-minus-ipsilateral amplitude differences $(\mu \mathrm{V})$ at electrode sites of interest

are at least in part characterized by late working memory stage response to distractors, rather than a hyper-selection of targets.

The visual search task employed here (Task A) found no evidence for an association between psychopathic personality traits and selective attention for targets. PPI-R scores were uncorrelated with RT or the amplitude (i.e., strength) of the target N2pc, a component that indexes the strength, timing, and reliability of visual-spatial selection (Luck \& Hillyard, 1994a; McDonald et al., 2013). Additionally, PPI-R scores did not moderate (influenced the relationship of) the correlation of RT with N2pc latency during visual search. PPI-R scores also were uncorrelated with target Ppc and CDA amplitudes. These components respectively index the value of salient features before selection (Christie et al., 2015; Jannati et al., 2013) and item manipulation within working memory (Gaspar \& McDonald, 2014; Jolicœur et al., 2008; Mazza et al., 2007; Vogel \& Machizawa, 2004). As such, there is no evidence in the current study that enhanced target response is the mechanism by which psychopathic personality traits impact visual attention.

This is not to say that visual-spatial attention abnormalities were unrelated to psychopathic personality traits. On the contrary, in the present study psychopathic impulsivity scores enhanced a potential neural indicator of distractor suppression. When participants focused on a central fixation point and ignored peripheral singleton distractors (Task B), higher SCI scores were correlated with greater positive amplitude of the CDA component. Although distractors failed to elicit the expected significant $\mathrm{P}_{\mathrm{D}}$ effect, the significant positive amplitude CDA to distractor singletons potentially indicates that visual-spatial suppression occurred at a working memory stage. This interpretation is based on recent studies reporting positive amplitude CDA components in response to lateralized distractors, an effect that was correlated with individual differences in visual working memory capacity (Feldmann-Wüstefeld \& Vogel, 2018; Fortier-Gauthier et al., 2012). Correlation of this component with SCI scores suggests a psychopathic impulsivity-related increase in distractor suppression within working memory.

Notably, a recent behavioral study conducted with an undergraduate student sample examined whether psychopathic traits measured with the PPI-R impact the processing of salient distractors during visual search (Hoppenbrouwers, Van der Stigchel, Sergiou, \& Theeuwes, 2016). This study found no 
Ppc

(75 - $125 \mathrm{~ms} ; \mathrm{O} 1 / \mathrm{O} 2)$

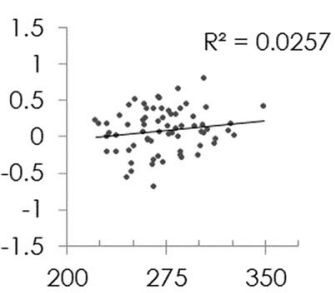

$\mathbf{P}_{\mathrm{D}}$

(250 - $310 \mathrm{~ms} ; \mathrm{PO} / \mathrm{PO}$ )
Early CDA

(350 - $410 \mathrm{~ms} ; \mathrm{PO} / \mathrm{PO} 8)$
Late CDA

(450 - 510 ms; PO7/PO8)
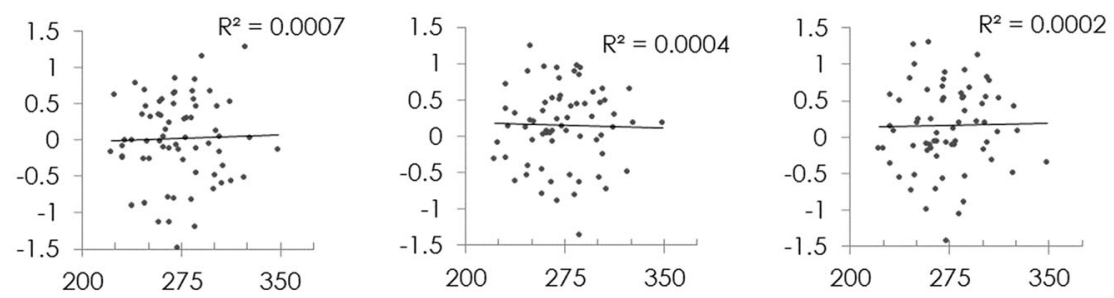

Total Score
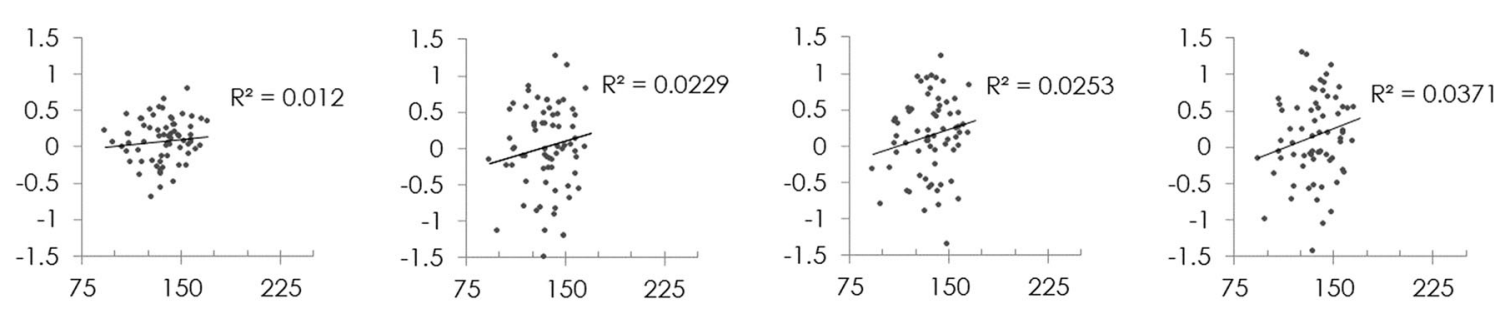

Self-centred Impulsivity
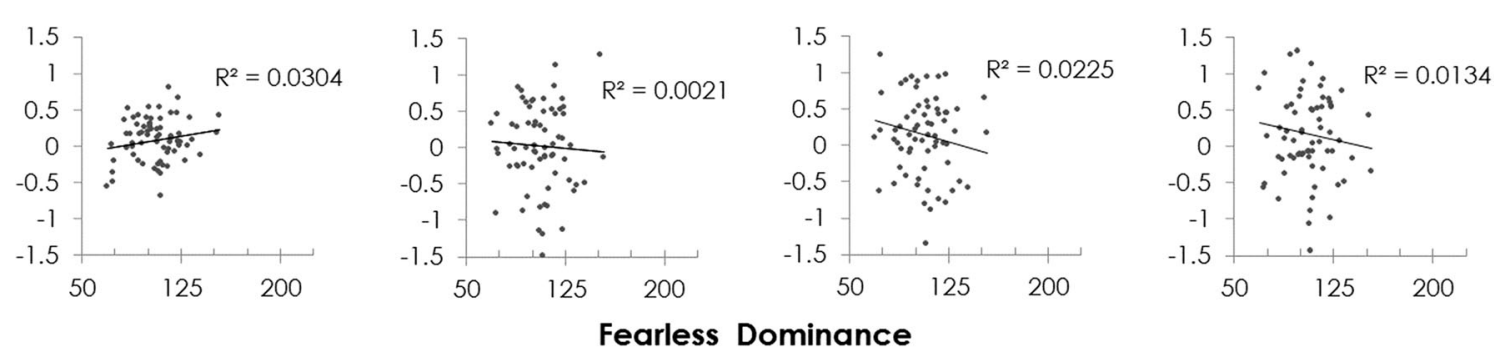

Fearless Dominance

Fig. 6 Fixation task (Task B), slow-response trials. Scatter plots of Psychopathic Personality Inventory-Revised total and factor scores by component grand average contralateral-minus-ipsilateral amplitude differences $(\mu \mathrm{V})$ at electrode sites of interest

significant covariance of RT with PPI scores (version 1 of the scale, Dutch translation), leading Hoppenbrouwers et al. to conclude that there was no behavioral evidence that psychopathic traits impact bottom-up processing of salient distractors. Similarly, in the current study there was no correlation of PPI-R total or factor scores with behavioral measures of distractor cost. However, the ERP data clearly suggest that visual-spatial processing of distractors is impacted by psychopathic impulsivity traits none the less.

The absence of a significant association between PPI-R scores and target-related Ppc, N2pc, or CDA components in the visual search task of the current study was notable given a recent publication of enhanced contingent capture for probes with target features (i.e., greater probe N2pc amplitude) amongst incarcerated offenders with high PCL-R scores (Krusemark et al., 2016). Such contingent capture effects reflect the influence of an endogenously held attention set that primes responding to target features, and so the results of Krusemark et al. indicate that psychopathic personality is associated with a top-down attention control process associated with target detection. The findings of the current investigation however suggest that these early attention enhancements do not translate into an advantage during the actual selection or evaluation of targets themselves, which would have been evidenced here by enhanced target Ppc, N2pc, or CDA components. This may reflect a key dissociable contribution of psychopathic traits to the actual goal-directed deployment of attention to targets (spatial selection) versus the establishment of a goal-directed attention set. Alternatively, whereas the current sample was predominantly composed of female undergraduate students assessed for psychopathic traits using the PPI-R, this may have resulted from differences in sample characteristics and the operation of scoring instruments (see Limitations, considerations, and directions below). Further investigation of visual search ERP components that integrate the target and distractor-related approaches used here, and the probe-related approach employed by Krusemark et al. with an incarcerated sample assessed using the PCL-R may elucidate this issue.

It also is worth considering the differential contributions of the two PPI-R factors, SCI and FD to the present results. The group level positive CDA component reported here was moderated by SCI, but not by FD or PPI-R total score. This aligns well with existing research examining differential 
contributions of affective-interpersonal versus impulsiveantisocial dimensions of psychopathy to selective attention and working memory. SCI is thought to capture the irresponsible lifestyle and externalizing traits that comprise the impulsive-antisocial domain of the PCL-R and shows good convergent validity with it and related measures (Benning et al., 2003; Kastner et al., 2012; Edens \& McDermott, 2010). Congruent with the current findings, previous research has linked problems with working memory, executive functioning, and response inhibition to the SCI factor of the PPI-R and similar scales in community samples (Carlson, Thái, \& McLarnon, 2009; Sadeh \& Verona, 2008; Sellbom \& Verona, 2007) and the impulsive-antisocial domain of the PCL-R of incarcerated samples (Baskin-Sommers, Wallace, MacCoon, Curtin, \& Newman, 2010). In contrast, early attention deficits have typically been associated with fearless dominance personality traits in community samples (Dvorak-Bertsch et al., 2009; Sadeh \& Verona, 2008) and the interpersonal-affective domain of the PCL-R in incarcerated samples (DvorakBertsch, Curtin, Rubinstein, \& Newman, 2009; Newman, Curtin, Bertsch, \& Baskin-Sommers, 2010; Sadeh \& Verona, 2008, 2012).

\section{Limitations, considerations, and future directions}

The absence of a significant $\mathrm{P}_{\mathrm{D}}$ component across the full sample and the lack of a correlation between behavioral performance and PPI-R scores in the fixation target task (Task B) presents a key issue for the interpretation of this study. The $P_{D}$ is consistently observed to index the goal-directed suppressing of salient distracting information. Instead, the presence of a positive amplitude CDA component was taken as indicative of a suppression mechanism similar to the $\mathrm{P}_{\mathrm{D}}$. Because the correlation of CDA amplitude and reaction time failed to reach significance, there are limits to this interpretation. However, it is in line with findings from a recent study of working memory encoding in which a positive amplitude CDA was correlated with individual difference in visual working memory capacity (Feldmann-Wüstefeld \& Vogel, 2018). This also begs the question as to why suppression was not observed during earlier visual encoding. It is possible that the absence of a clear $\mathrm{P}_{\mathrm{D}}$ effect during the fixation task was due to the nature of the relationship between the target and distractor. The strongest $\mathrm{P}_{\mathrm{D}}$ effects observed in previous literature (Gaspar \& McDonald, 2014; McDonald et al., 2013; Sawaki et al., 2012) have been for tasks employing a "dual singleton" format in which both the target and distractor locations vary from trial-to-trial, and both singletons vary on the same feature dimension (e.g., a red target and yellow distractor in a group of green fillers). In the current study, the $\mathrm{P}_{\mathrm{D}}$ may have been obscured by the use of a luminance target and a shape singleton. Furthermore, whereas the component is believed to assist deployment of spatial attention to targets during search, the use of a spatially invariant target likely reduced the capacity of the fixation task to evoke a strong $\mathrm{P}_{\mathrm{D}}$.

The design of the experimental tasks employed in this study also presents some challenges in terms of relating results to the existing psychopathy response modulation literature. In particular, studies of the response modulation hypothesis have focused on the role of attention set in limiting the access of peripheral information into awareness (Baskin-Sommers \& Newman, 2014; Glass \& Newman, 2006; Newman \& Baskin-Sommers, 2012; Patterson \& Newman, 1993; Wolf et al., 2012). Furthermore, ERP studies of attention abnormalities in psychopathic personality have typically examined early fronto-central effects (Baskin-Sommers et al., 2012; Hamilton et al., 2014). In contrast, our visual search tasks required the use of spatial attention mechanisms to guide visual search and measured the influence of psychopathic personality traits on these effects using lateralized ERP components traditionally measured over occipital and posterior scalp.

The current results provide insight into visual cortex mechanisms that may underlie spatial attention effects observed in studies of the response modulation theory (Hamilton et al., 2014; Hiatt et al., 2004; Zeier et al., 2009) and do much to situate attention bottleneck proposals of psychopathy within the expansive field of visual search ERP research. However, the working memory effects observed here may related better to abnormalities proposed in other models of psychopathy. Recently, the impaired integration framework (Hamilton, Hiatt Racer, \& Newman, 2015) has been proposed as a possible unifying account of attention-based and affect-based theories of psychopathic personality. Within this model, diminished perceptual integration capacity is argued to be the fundamental deficit underlying both the cognitive and emotional dysfunctions found in psychopathy. Individuals high in psychopathy are thought to have difficulty rapidly binding the components of complex, multidimensional stimuli. As a result, even under low perceptual load conditions, they have limited capacity to allocate resources to stimuli beyond their current focus of attention (Hamilton et al., 2015). Following this logic, it is possible that the increased working memory activity observed in the current study reflected a relative increase in perceptual load for participants higher in psychopathic personality traits.

Additionally, while this study elucidates the selective attention abnormalities associated with high levels of psychopathic traits, an important caveat must be reiterated. The sample presented here was a predominantly female group of typical undergraduate students assessed with the PPI-R, and few if any would qualify as psychopathic. In contrast, many previous studies of the response modulation hypothesis, particularly Krusemark et al.'s (2016) ERP study finding enhanced N2pc 
response to target-like probes during visual search, have been conducted with incarcerated samples assessed with the PCL$\mathrm{R}$. There remains considerable debate about whether psychopathic personality is best conceptualized as a syndrome-a cluster of symptoms that are together indicative of an underlying pathology — or an interpersonal impact condition-a compound of uncorrelated traits from which emerges a socially distinctive impression (Lilienfeld, 2013). This debate is reflected in the design of many psychopathy measures. For example, although the PCL-R is intended to assess psychopathy as a syndrome, psychopathy as measured by the PPI-R is non-syndromal (Lilienfeld, 2013). If severe presentations of psychopathy are indeed instances of a syndrome, then this may explain why no N2pc effects were seen to targets in the current study or why no correlations were observed between PPI-R scores and behavioral measures. However, if psychopathic personality is instead an emergent trait of social relevance, then the findings observed here may be replicated in offender samples. In either case, it might be expected that the undergraduates in the current study possessed greater executive functioning than the participants in many existing publications examining psychopathy and attention. This highlights the need for research specifically examining distractor suppression and psychopathy in incarcerated participants.

Finally, it is unclear how these ERP effects would be influenced by the presentation of inhibitory cues as peripheral stimuli (e.g., a stop-signal task). The current data provided electrophysiological evidence that task-irrelevant distractors may have been suppressed more from working memory as SCI scores increased. Within the response modulation theory, a logical extension of this research would be to examine whether findings are replicated with peripheral distractors that also function as stop-signals or if the strength of a distractor ERP mediates propensity to perseverate despite punishment contingencies.

These limitations notwithstanding, this study expands the attention bottleneck proposal of psychopathic personality by identifying two critical characteristics of visual-cortical response to salient targets and distractors in an undergraduate sample. First, intentional deployment of selective spatial attention to salient targets was not altered by psychopathic personality, discounting an explanation premised solely on enhanced or hyper-selective attention. Second, psychopathic impulsivity traits were positively associated with increased suppression of distractors at a later stage typically associated with working memory processes. These conclusions dovetail to a possible neurophysiological explanation of the attention abnormality proposed within the response modulation theory and similar attention-based accounts of psychopathy. Resistance to peripheral, task-irrelevant information seems to arise from greater suppression of external content, rather than heightened selection of task relevant content. What remains to be seen is whether this pattern of attention is replicated when the salience of task-irrelevant stimuli is defined by reinforcement and punishment learning, their preexisting affective value, or their utility as task-relevant inhibitory cues.

None of the data for the experiments reported are available in an online repository, and none of the experiments were preregistered.

Author Note Patrick L. Carolan is now at the Department of Psychology, Saint Mary's University, Halifax. Mario Liotti is now at the Department of Developmental and Social Psychology, and the Padua Neuroscience Center, University of Padua, Italy.

This research was supported by grants from the National Science and Engineering Research Council (NSERC) of Canada (grant \# 365026), and the Canada Foundation for Innovation (CFI) to Mario Liotti. Preliminary findings were presented at the $24^{\text {th }}$ annual meeting of the Cognitive Neuroscience Society, San Francisco, California, 2017.

\section{References}

Anderson, N. E., \& Stanford, M. S. (2012). Demonstrating emotional processing differences in psychopathy using affective ERP modulation. Psychophysiology, 49(6), 792-806. https://doi.org/10.1111/j. 1469-8986.2012.01369.x

Baron, R. M., \& Kenny, D. A. (1986). The moderator-mediator variable distinction in social psychological research: Conceptual, strategic, and statistical considerations. Journal of Personality and Social Psychology, 51(6), 1173. https://doi.org/10.1037/00223514.51.6.1173

Baskin-Sommers, A., Curtin, J. J., Li, W., \& Newman, J. P. (2012). Psychopathy-Related Differences in Selective Attention Are Captured by an Early Event-Related Potential. Personality Disorders, 3(4), 370-378. https://doi.org/10.1037/a0025593

Baskin-Sommers, A. R., \& Newman, J. P. (2014). Psychopathic and externalizing offenders display dissociable dysfunctions when responding to facial affect. Personality Disorders: Theory, Research, and Treatment, 5(4), 369-379. https://doi.org/10.1037/ per0000077

Baskin-Sommers, A. R., Wallace, J. F., MacCoon, D. G., Curtin, J. J., \& Newman, J. P. (2010). Clarifying the Factors that Undermine Behavioral Inhibition System Functioning in Psychopathy. Personality Disorders, 1(4), 203-217. https://doi.org/10.1037/ a0018950

Benning, S. D., Patrick, C. J., Hicks, B. M., Blonigen, D. M., \& Krueger, R. F. (2003). Factor Structure of the Psychopathic Personality Inventory: Validity and Implications for Clinical Assessment. Psychological Assessment, 15(3), 340-350. https://doi.org/10. 1037/1040-3590.15.3.340

Burra, N., Barras, C., Coll, S. Y., \& Kerzel, D. (2016). Electrophysiological evidence for attentional capture by irrelevant angry facial expressions. Biological Psychology, 120, 69-80. https:// doi.org/10.1016/j.biopsycho.2016.08.008

Burra, N., \& Kerzel, D. (2014). The distractor positivity (Pd) signals lowering of attentional priority: Evidence from event-related potentials and individual differences. Psychophysiology, 51, 685-696. https://doi.org/10.1111/psyp.12215 
Carlson, S. R., Thái, S., \& McLarnon, M. E. (2009). Visual P3 amplitude and self-reported psychopathic personality traits: Frontal reduction is associated with self-centered impulsivity. Psychophysiology, 46(1), 100-113. https://doi.org/10.1111/j.1469-8986.2008.00756.x

Carolan, P. L., Jaspers-Fayer, F., Asmaro, D. T., Douglas, K. S., \& Liotti, M. (2014). Electrophysiology of blunted emotional bias in psychopathic personality. Psychophysiology, 51(1), 36-41. https://doi.org/ 10.1111/psyp. 12145

Christie, G. J., Livingstone, A. C., \& McDonald, J. J. (2015). Searching for Inefficiency in Visual Search. Journal of Cognitive Neuroscience, 27(1), 46-56. https://doi.org/10.1162/jocn_a 00716

Cohen, J. (1992). A power primer. Psychological bulletin, 112(1), 155 159. https://doi.org/10.1037/0033-2909.112.1.155

Cohen, J., Cohen, P., West, S. G., \& Aiken, L. S. (2003). Applied multiple regression/correlation analysis for the behavioral sciences (3rd ed.). Routledge.

Di Lollo, V., Enns, J. T., \& Rensink, R. A. (2000). Competition for consciousness among visual events: The psychophysics of reentrant visual processes. Journal of Experimental Psychology: General, 129(4), 481-507. https://doi.org/10.1037/0096-3445.129.4.481

Dvorak-Bertsch, J. D., Curtin, J. J., Rubinstein, T. J., \& Newman, J. P. (2009). Psychopathic traits moderate the interaction between cognitive and affective processing. Psychophysiology, 46(5), 913-921. https://doi.org/10.1111/j.1469-8986.2009.00833.x

Eimer, M., \& Kiss, M. (2008). Involuntary attentional capture is determined by task set: evidence from event-related brain potentials. Journal of Cognitive Neuroscience, 20(8), 1423-1433. https://doi. org/10.1162/jocn.2008.20099

Eimer, M., \& Kiss, M. (2010). The top-down control of visual selection and how it is linked to the N2pc component. Acta Psychologica, 135(2), 100-102. https://doi.org/10.1016/j.actpsy.2010.04.010

Feldmann-Wüstefeld, T., \& Vogel, E. K. (2018). Neural Evidence for the Contribution of Active Suppression During Working Memory Filtering. Cerebral Cortex, 29(2), 529-543. https://doi.org/10.1093/ cercor/bhx336

Fortier-Gauthier, U., Moffat, N., Dell'Acqua, R., McDonald, J. J., \& Jolicœur, P. (2012). Contralateral cortical organisation of information in visual short-term memory: Evidence from lateralized brain activity during retrieval. Neuropsychologia, 50(8), 1748-1758. https://doi.org/10.1016/j.neuropsychologia.2012.03.032

Gaspar, J. M., Christie, G. J., Prime, D. J., Jolicœur, P., \& McDonald, J. J. (2016). Inability to suppress salient distractors predicts low visual working memory capacity. Proceedings of the National Academy of Sciences of the United States of America, 113(13), 3693-3698. https://doi.org/10.1073/pnas.1523471113

Gaspar, J. M., \& McDonald, J. J. (2014). Suppression of Salient Objects Prevents Distraction in Visual Search. Journal of Neuroscience, 34(16), 5658-5666. https://doi.org/10.1523/JNEUROSCI.4161-13. 2014

Glass, S. J., \& Newman, J. P. (2006). Recognition of facial affect in psychopathic offenders. Journal of Abnormal Psychology, 115(4), 815-820. https://doi.org/10.1037/0021-843X.115.4.815

Hamilton, R. K. B., Hiatt Racer, K., \& Newman, J. P. (2015). Impaired integration in psychopathy: A unified theory of psychopathic dysfunction. Psychological Review, 122(4), 770-791. https://doi.org/ 10.1037/a0039703

Hamilton, R. K. B., Baskin-Sommers, A. R., \& Newman, J. P. (2014). Relation of frontal N100 to psychopathy-related differences in selective attention. Biological Psychology, 103, 107-116. https://doi. org/10.1016/j.biopsycho.2014.08.012

Hare, R. D. (2003). Manual for the Revised Psychopathy Checklist (2nd ed.). Multi-Health Systems.

Hiatt, K. D., Schmitt, W. A., \& Newman, J. P. (2004). Stroop tasks reveal abnormal selective attention among psychopathic offenders. Neuropsychology, 18(1), 50-59. https://doi.org/10.1037/08944105.18.1.50
Hickey, C., Di Lollo, V., \& McDonald, J. J. (2009). Electrophysiological indices of target and distractor processing in visual search. Journal of Cognitive Neuroscience, 21(4), 760-775. https://doi.org/10.1162/ jocn.2009.21039

Hopfinger, J. B., \& Ries, A. J. (2005). Automatic versus contingent mechanisms of sensory-driven neural biasing and reflexive attention. Journal of Cognitive Neuroscience, 17(8), 13411352. https://doi.org/10.1162/0898929055002445

Hoppenbrouwers, S. S., Van der Stigchel, S., Sergiou, C. S., \& Theeuwes, J. (2016). Top-down attention and selection history in psychopathy: Evidence from a community sample. Journal of Abnormal Psychology, 125(3), 435-441. https://doi.org/10.1037/abn0000133

Jannati, A., Gaspar, J. M., \& McDonald, J. J. (2013). Tracking target and distractor processing in fixed-feature visual search: Evidence from human electrophysiology. Journal of Experimental Psychology: Human Perception and Performance, 39(6), 1713-1730. https:// doi.org/10.1037/a0032251

Jolicœur, P., Brisson, B., \& Robitaille, N. (2008). Dissociation of the $\mathrm{N} 2 \mathrm{pc}$ and sustained posterior contralateral negativity in a choice response task. Brain Research, 1215, 160-172. https://doi.org/10. 1016/j.brainres.2008.03.059

Jung, T. P., Humphries, C., Lee, T. W., Makeig, S., McKeown, M. J., Iragui, V., \& Sejnowski, T. J. (1998a). Extended ICA removes artifacts from electroencephalographic recordings. In Advances in neural information processing systems (pp. 894-900). Retrieved from https://papers.nips.cc/paper/1343-extended-ica-removes-artifactsfrom-electroencephalographic-recordings.pdf

Jung, T. P., Humphries, C., Lee, T. W., Makeig, S., McKeown, M. J., Iragui, V., \& Sejnowski, T. J. (1998b). Removing electroencephalographic artifacts: comparison between ICA and PCA. Neural Networks for Signal Processing VIII. Proceedings of the 1998 IEEE Signal Processing Society Workshop (Cat. No.98TH8378), 63-72. https://doi:10.1109/NNSP.1998.710633

Kastner, R. M., Sellbom, M., \& Lilienfeld, S. O. (2012). A comparison of the psychometric properties of the psychopathic personality inventory full-length and short-form versions. Psychological Assessment, 24(1), 261-267. https://doi.org/10.1037/a0025832

Krusemark, E. A., Kiehl, K. A., \& Newman, J. P. (2016). Endogenous attention modulates early selective attention in psychopathy: An ERP investigation. Cognitive, Affective, \& Behavioral Neuroscience, 16(5), 779-788. https://doi.org/10.3758/s13415016-0430-7

Lilienfeld, S. O. (2013). Is psychopathy a syndrome? Commentary on Marcus, Fulton, and Edens. Personality Disorders: Theory, Research, and Treatment, 4(1), 85-86. https://doi.org/10.1037/ a0027544

Lilienfeld, S. O., \& Widows, M. R. (2005). PPI-R: Psychopathic personality inventory revised: Professional Manual. Psychological Assessment Resources, Incorporated.

Livingstone, A. C., Christie, G. J., Wright, R. D., \& McDonald, J. J. (2017). Signal enhancement, not active suppression, follows the contingent capture of visual attention. Journal of Experimental Psychology: Human Perception and Performance, 43(2), 219 224. https://doi.org/10.1037/xhp0000339

Luck, S. J. (2014). An Introduction to the Event-Related Potential Technique. MIT Press.

Luck, S. J., \& Hillyard, S. A. (1994a). Electrophysiological correlates of feature analysis during visual search. Psychophysiology, 31(3), 291308. https://doi.org/10.1111/j.1469-8986.1994.tb02218.x

Luck, S. J., \& Hillyard, S. A. (1994b). Spatial filtering during visual search: Evidence from human electrophysiology. Journal of Experimental Psychology: Human Perception and Performance, 20(5), 1000-1014. https://doi.org/10.1037/0096-1523.20.5.1000

Luck, S. J., \& Kappenman, E. S. (2013). The Oxford Handbook of EventRelated Potential Components. Oxford University Press. 
Mazza, V., Turatto, M., Umiltà, C., \& Eimer, M. (2007). Attentional selection and identification of visual objects are reflected by distinct electrophysiological responses. Experimental Brain Research. Experimentelle Hirnforschung. Experimentation Cerebrale, 181(3), 531-536. https://doi.org/10.1007/s00221-007-1002-4

McDonald, J. J., Green, J. J., Jannati, A., \& Di Lollo, V. (2013). On the electrophysiological evidence for the capture of visual attention. Journal of Experimental Psychology: Human Perception and Performance, 39(3), 849-860. https://doi.org/10.1037/a0030510

Medina, A. L., Kirilko, E., \& Grose-Fifer, J. (2016). Emotional processing and psychopathic traits in male college students: An eventrelated potential study. International Journal of Psychophysiology, 106, 39-49. https://doi.org/10.1016/j.jpsycho.2016.06.004

Newman, J. P., \& Baskin-Sommers, A. R. (2012). Early selective attention abnormalities in psychopathy: Implications for self-regulation. In Cognitive neuroscience of attention (2nd ed., pp. 421-440). Guilford Press.

Newman, J. P., Curtin, J. J., Bertsch, J. D., \& Baskin-Sommers, A. R. (2010). Attention moderates the fearlessness of psychopathic offenders. Biological Psychiatry, 67(1), 66-70. https://doi.org/10. 1016/j.biopsych.2009.07.035

Newman, J. P., \& Kosson, D. S. (1986). Passive avoidance learning in psychopathic and nonpsychopathic offenders. Journal of Abnormal Psychology, 95(3), 252-256. https://doi.org/10.1037/0021-843X. 95.3.252

Oostenveld, R., Fries, P., Maris, E., \& Schoffelen, J. M. (2011). FieldTrip: Open Source Software for Advanced Analysis of MEG, EEG, and Invasive Electrophysiological Data. Computational Intelligence and Neuroscience, 2011, 1-9. https://doi.org/10.1155/2011/156869

Patterson, C. M., \& Newman, J. P. (1993). Reflectivity and learning from aversive events: Toward a psychological mechanism for the syndromes of disinhibition. Psychological Review, 100(4), 716. https://doi.org/10.1037/0033-295X.100.4.716

Ray, J., Weir, J. W., Poythress, N. G., \& Rickelm, A. (2011). Correspondence between the psychopathic personality inventory and the psychopathic personality inventory-revised. Criminal Justice and Behavior, 38(4), 375-385. https://doi.org/10.1177/ 0093854811398178

Sadeh, N., \& Verona, E. (2008). Psychopathic personality traits associated with abnormal selective attention and impaired cognitive control. Neuropsychology, 22(5), 669-680. https://doi.org/10.1037/ a0012692

Sadeh, N., \& Verona, E. (2012). Visual complexity attenuates emotional processing in psychopathy: Implications for fear-potentiated startle deficits. Cognitive, Affective, \& Behavioral Neuroscience, 12(2), 346-360. https://doi.org/10.3758/s13415-011-0079-1

Sawaki, R., Geng, J. J., \& Luck, S. J. (2012). A common neural mechanism for preventing and terminating the allocation of attention. The Journal of Neuroscience: The Official Journal of the Society for Neuroscience, 32(31), 10725-10736. https://doi.org/10.1523/ JNEUROSCI.1864-12.2012

Sellbom, M., \& Verona, E. (2007). Neuropsychological correlates of psychopathic traits in a non-incarcerated sample. Journal of Research in Personality, 41(2), 276-294. https://doi.org/10.1016/j.jrp.2006.04. 001

Theeuwes, J. (2010). Top-down and bottom-up control of visual selection. Acta Psychologica, 135(2), 77-99. https://doi.org/10.1016/j. actpsy.2010.02.006

Uzieblo, K., Verschuere, B., Van den Bussche, E., \& Crombez, G. (2010). The Validity of the Psychopathic Personality Inventory - Revised in a Community Sample. Assessment, 17(3), 334-346. doi: https://doi. org/10.1177/1073191109356544

Vogel, E. K., \& Luck, S. J. (2000). The visual N1 component as an index of a discrimination process. Psychophysiology, 37(2), 190203. https://doi.org/10.1111/1469-8986.3720190

Vogel, Edward K., \& Machizawa, M. G. (2004). Neural activity predicts individual differences in visual working memory capacity. Nature, 428(6984), 748-751. https://doi.org/10.1038/nature02447

Wolf, R. C., Warren, C. M., Carpenter, R. W., Zeier, J. D., BaskinSommers, A. R., \& Newman, J. P. (2012). Reduced Susceptibility to the Attentional Blink in Psychopathic offenders: Implications for the Attention Bottleneck Hypothesis. Neuropsychology, 26(1), 102109. https://doi.org/10.1037/a0026000

Wolfe, J. M. (2007). Guided search 4.0. Integrated Models of Cognitive Systems, 99-119. https://doi.org/10.1093/acprof:oso/ 9780195189193.003.0008

Wolfe, J. M., \& Horowitz, T. S. (2004). What attributes guide the deployment of visual attention and how do they do it? Nature Reviews Neuroscience, 5(6), 495-501. https://doi.org/10.1038/nrn1411

Zeier, J. D., Maxwell, J. S., \& Newman, J. P. (2009). Attention Moderates the Processing of Inhibitory Information in Primary Psychopathy. Journal of Abnormal Psychology, 118(3), 554-563. https://doi.org/ $10.1037 / \mathrm{a} 0016480$

Publisher's note Springer Nature remains neutral with regard to jurisdictional claims in published maps and institutional affiliations. 